\title{
Tai Chi Chuan for Subjective Sleep Quality: A Systematic Review and Meta-Analysis of Randomized Controlled Trials
}

\author{
Yuhao Si $\mathbb{D}^{1,2}$ Cenyi Wang $\mathbb{D}^{1,3}$ Heng Yin ${ }^{2},{ }^{4}$ Jinghui Zheng, ${ }^{5}$ Yang Guo, ${ }^{1}$ Guihua Xu, ${ }^{6}$ \\ and Yong $M a \mathbb{B}^{7,8}$ \\ ${ }^{1}$ The First School of Clinical Medicine, Nanjing University of Chinese Medicine, Nanjing 210023, China \\ ${ }^{2}$ Rangos School of Health Sciences, Duquesne University, Pittsburgh 15282, USA \\ ${ }^{3}$ School of Physical Education and Sports Science, Soochow University, Suzhou 215021, China \\ ${ }^{4}$ Department of Traumatology \& Orthopedics, Wuxi Affiliated Hospital of Nanjing University of Chinese Medicine, \\ Wuxi 214071, China \\ ${ }^{5}$ Department of Cardiology, Ruikang Hospital Affiliated to Guangxi University of Chinese Medicine, Nanning 530011, China \\ ${ }^{6}$ School of Nursing, Nanjing University of Chinese Medicine, Nanjing 210023, China \\ ${ }^{7}$ Department of Traumatology \& Orthopedics, Affiliated Hospital of Nanjing University of Chinese Medicine, \\ Nanjing 210029, China \\ ${ }^{8}$ College of Basic Medicine, Nanjing University of Chinese Medicine, Nanjing 210023, China
}

Correspondence should be addressed to Yong Ma; zhongyimayong@126.com

Received 17 March 2020; Revised 10 May 2020; Accepted 9 June 2020; Published 11 August 2020

Academic Editor: Christopher Worsnop

Copyright (c) 2020 Yuhao Si et al. This is an open access article distributed under the Creative Commons Attribution License, which permits unrestricted use, distribution, and reproduction in any medium, provided the original work is properly cited.

Background. This review aims to investigate the efficacy of Tai Chi Chuan on subjective sleep quality among adults. Methods. We systematically searched PubMed, Embase, Cochrane Library, Scopus, CNKI (China National Knowledge Infrastructure), and the Wanfang Database from their inception to August 2019 and identified 25 eligible studies that were published in both English and Chinese. Results. 24 out of 25 studies were identified to be high-quality studies according to the PEDro scale. The pooled results confirmed that Tai Chi Chuan elicited moderate improvements in subjective sleep quality (SMD $=-0.512,95 \%$ CI $[-0.767$, -0.257], $P<0.001)$. Notably, Tai Chi Chuan yielded more significant effects on sleep quality among the healthy population $(\mathrm{SMD}=-0.684,95 \% \mathrm{CI}[-1.056,-0.311], P<0.001)$ than the clinical population $(\mathrm{SMD}=-0.395,95 \%$ CI $[-0.742,-0.047]$, $P=0.026)$ and more benefits among the Asian population $(\mathrm{SMD}=-0.977,95 \% \mathrm{CI}[-1.446,-0.508], P<0.001)$ than the American population (SMD $=-0.259,95 \% \mathrm{CI}[-0.624,0.105], P=0.164)$. After controlling the methodological quality of studies, it has been noted that Asians could achieve the most significant sleep-promoting benefit when Tai Chi Chuan was practiced between 60 and 90 min per session. Conclusions. Available data implied that subjective sleep quality was improved via Tai Chi training, but more thorough studies must be executed to ascertain our findings and optimize Tai Chi practices accordingly toward various populations.

\section{Introduction}

Sleep plays a critical role in the general health of human beings, accounting for almost $1 / 3$ of our lifetimes [1]. Adequate and comfortable sleep is important for motivation and enthusiasm yielding high energy and a happy mood along with the ability to handle daytime routine tasks. However, poor sleep quality has become a universal issue in modern society, causing insidious physical and psychological disorders in both healthy individuals as well as clinical patients $[2,3]$. Recent epidemiological investigations indicated that approximately $25 \%$ of adults had sleep complaints, $10 \%-15 \%$ experienced insomnia symptoms along with daytime consequences, and 6\%-10\% met the diagnostic criteria for insomnia disorder [4-9]. Additionally, some indirect consequences caused by poor sleep quality, such as 
work absenteeism and reduced productivity, may also lead to increased economic and social burdens. Despite the pervasiveness and burden of the issue, poor sleep quality is usually overlooked and untreated due to evaluation and management barriers. It is estimated that only approximately $15 \%$ of adults with diagnosed insomnia or sleep complaints attempt to seek professional consultation [10].

Complementary and alternative approaches have been employed to mitigate sleep disorders for a long time, among which physical exercise draws increasing attention in recent years [11]. As a type of physical exercise rooted in traditional Chinese medicine, Tai Chi Chuan has been practiced to invigorate both physical and mental health and prevent diseases for hundreds of years in China [12]. Nowadays, Tai Chi Chuan is drawing legions of practitioners and followers in western countries [13]. It consists of slow movements, weight shifting, concentration, and meditation. Such movements are thought to improve multiple aspects of body health such as control of breath, calming the mind, increasing musculoskeletal strength, and enhancing balance as well as functions of the autonomic nervous and immune systems $[14,15]$. Numerous systematic reviews have been directed as a means of providing high-quality evidence of the role of Tai Chi Chuan in alleviating chronic conditions. Some recent reviews have investigated and supported the use of Tai Chi Chuan exercise for chronic conditions, such as cancer-related symptoms [16], cognition impairment [17], type 2 diabetes mellitus [18], stroke rehabilitation [19], coronary heart disease [20], heart failure [21], osteoporosis [22], hypertension [23], chronic musculoskeletal pain [24], chronic obstructive pulmonary disease [25], osteoarthritis [26], and rheumatoid arthritis [27]. Notably, Tai Chi Chuan training could also be efficacious in reducing fall incidence and maintaining and enhancing lower limb proprioception and gait ability for the aged [28]. Overall, the aforementioned reviews reported moderate to strong evidence of Tai Chi Chuan's positive effects on related chronic conditions. However, the application of Tai Chi Chuan on sleep quality seems to dictate varying results.

Some previous systematic reviews evaluated the effectiveness of Tai Chi Chuan (may be presented as a type of mind-body therapies or meditative movements) on sleep quality [29-33]. However, certain limitations were discovered in the previous systematic reviews. Firstly, several systematic reviews meta-analyzed numerous trials regarding mind-body exercises (e.g., Qigong, Yoga, and Tai Chi Chuan), but Tai Chi Chuan only accounted for a small portion of those included trials, implying a lack of independent assessments and weak evidence. This is not to mention that people with various physical conditions or diseases can respond to Tai Chi exercise in different ways, and no analysis was performed based on different types of physical conditions. Finally, some trials of Tai Chi Chuan published in China may potentially have been missed because of the language barriers during the retrieval process. We conducted a systematic review in 2014 to solely investigate the impact of Tai Chi exercise on sleep quality among older adults [34]. In our previous study, five randomized controlled trials (RCTs) were pooled in the meta-analysis, which yielded weak evidence showing a beneficial impact of Tai Chi exercise on self-rated sleep quality in healthy older adults. Additionally, due to a lack of evidence, the guidelines presented by the American College of Physicians and European Sleep Research Society both suggested that it is necessary for complementary and alternative therapy or exercise to undergo further assessment as a means to judge its usefulness in the treatment of insomnia $[35,36]$. Therefore, for further study, the current review aims to evaluate the effects of Tai Chi Chuan for sleep quality while employing more updated rigorous RCTs that covered both healthy and clinical populations. Sleep quality is assessed either subjectively through self-report or by objective measures. Nonetheless, perceived sleep quality does not necessarily correspond with objective sleep measures. The Pittsburgh Sleep Quality Index (PSQI) is one of the most frequently used instruments for measuring subjective sleep quality and has excellent psychometric properties. Therefore, we applied the outcome of subjective sleep quality in this review due to its evaluation convenience and clinical significance [37].

\section{Methods}

2.1. Search Strategy. This review was executed according to the guidance in the "Preferred Reporting Items for Systematic Reviews and Meta-Analyses" (PRISMA) statement [38]. The protocol was prospectively registered in the International Prospective Register of Systematic Reviews (PROSPERO) database in March 2019 (registration number: CRD42019129782) and was published in November 2019 [39].

We designed a broad literature search strategy aiming to identify all the eligible RCTs published in the English and Chinese language in peer-reviewed journals. Six electronic databases, including PubMed, Embase, Cochrane Library, Scopus, CNKI, and the Wanfang Database, were searched to identify trials relating to the effectiveness of Tai Chi Chuan for sleep quality from their inception to August 2019. The following search terms were employed in the English databases (PubMed, Embase, Cochrane Library, and Scopus): Tai Chi, Taiji, Tai Chi Chuan, shadowboxing, taijiquan, sleep, sleep quality, sleep complaints, sleep problems, sleep disorders, sleep disturbance, and insomnia. The equivalent Chinese search terms were utilized in Chinese databases. A manual search was also implemented at the library of Duquesne University and the Nanjing University of Chinese Medicine in the event that there were any missing eligible articles.

2.2. Study Selection. Studies involved in this review met the following criteria: (a) trial assessed the effects of Tai Chi Chuan for sleep quality; (b) the study design was RCT; (c) participants were adults; (d) the participants practiced Tai Chi Chuan in the experimental group and did not practice Tai Chi Chuan in the control group; (e) the outcome was measured through the PSQI scale; (f) the trial was published in English or Chinese. Studies that had any of the following 
criteria were excluded: (a) trials that used comprehensive interventions, such as Tai Chi Chuan with Chinese oral medicine or acupuncture; (b) data were unavailable to extract and calculate whether or not the corresponding author was contacted successfully.

Four reviewers were divided into two pairs, and each pair independently (Guo and Zheng; Si and Wang) screened all titles and abstracts in the initial search. After removing duplicated and unrelated articles, the lists of possibly relevant articles from the two pairs were checked against each other by another reviewer (Xu). The fifth reviewer (Xu) compared the results of two pairs and determined a preliminary list. The full-text reading and evaluation of articles from the preliminary list were implemented by two reviewers (Wang and Guo) following the predefined inclusion/ exclusion criteria. Any disagreement was discussed by the two reviewers to reach a consensus. If the disagreement remained unresolved, a final decision would be made by a third reviewer $(\mathrm{Ma})$.

2.3. Data Extraction and Quality Assessment. The process of data extraction was completed by two reviewers ( $\mathrm{Si}$ and Wang) and double-checked by a third reviewer (Ma). Subject characteristics and study designs were recorded in a Microsoft Excel document, including information such as first author, publication year, country, average age, geographic population, sample size, physical condition, duration and follow-up, frequency, intensity, Tai Chi Chuan style, control intervention, sleep quality measurement, baseline and outcome data, and adverse events. If such information was not reported in the article, we emailed the corresponding author to request for relevant materials.

Two researchers ( $\mathrm{Si}$ and $\mathrm{Ma}$ ) evaluated the methodological quality of the included studies through using the Physiotherapy Evidence Database (PEDro) scale. The PEDro scale consists of 11 items, and each item is rated from 0 to 10 points. A study with 6 points or more was identified as a high-quality study. Several previous studies recommended the PEDro scale to assess the quality of RCTs (mainly in the area of physical therapy) for systematic reviews because of its high validity and reliability [40-43].

\subsection{Statistical Analysis}

2.4.1. Effect Size Calculation. Sleep quality outcomes, in terms of mean and standard deviations (SD), either reported in the study or those that could be obtained from the corresponding author, were included in the meta-analyses. We utilized the changes from baseline scores for continuous data due to inconsistent baselines of eligible trials, being presented as the mean and SD. Moreover, the global PSQI (SD ranged from 0.44 to 5.78) from each study was the score of self-assessment with inevitable confounding factors, and the standardized mean difference (SMD) was used to provide robust evaluation over the unstandardized mean difference in various statistical situations [44]. According to Cohen [45], SMD is also known as the effect size (ES) and can be interpreted by large $(\mathrm{ES} \geq 0.8)$, moderate
$(0.5 \leq \mathrm{ES}<0.8)$, small $(0.2 \leq \mathrm{ES}<0.5)$, and trivial $(\mathrm{ES}<0.2)$. The heterogeneity was evaluated using the chi-square and $I^{2}$ test, which describes the percentage of variability in the effect estimates. $I^{2}$ of $0 \%, 25 \%, 50 \%$, and $75 \%$ implied nil, mild, moderate, and severe heterogeneity, respectively [46]. We chose a random-effects model to be applied for the metaanalysis because the expected diversity of involved subjects and interventions might lead to the possible existence of heterogeneity.

2.4.2. Subgroup Analysis. We conducted two preplanned subgroup analyses to explore the potential explanation for heterogeneity and to test the effects of Tai Chi exercise on sleep quality according to the varying situations. One subgroup analysis was administered based on the different physical conditions of the included adults (good health, stroke, fibromyalgia, cancer, arthritis, depression, chronic kidney disease, and heart disease). The other one was performed in accordance with geographic populations (Asian, American, and European) in the trials that enrolled healthy individuals.

2.4.3. Meta-Regression and Additive Models. The source of heterogeneity was explored utilizing a random-effects metaregression. Some characteristics of the recruited participants, as well as the study design and execution, were also explored in addition to the Tai Chi exercise movements. Specifically, aspects such as average age, sample size, control intervention (treated and nontreated intervention), physical condition (healthy and illness), disease (good health, stroke, fibromyalgia, cancer, depression, arthritis, chronic kidney disease, and heart disease), supervision (supervised, unsupervised, and both), methodological quality of trials (PEDro score), Tai Chi Chuan style (Yang style, Chen style, and Tai Chi Ball), treatment duration (week), training intensity (min/session), frequency (times/week), and geographic population (American, Asian, and European) were entered as variables. All these covariables were input and analyzed one by one. The final covariables were selected depending on their impact on sleep quality response to Tai Chi exercise, which were tested by the statistical significance of $P<0.05$ combined with adjusted $R^{2}$. For the change of PSQI scores, additive models were yielded from all the possible combinations of the final covariables to determine the most significant potential sleep-promoting effects from Tai Chi Chuan. Significant continuous variables such as training intensity (each class: 30-60 $\mathrm{min} ; 60-90 \mathrm{~min}$; 90-120 min) and methodological quality of trials (lowest tier, $\leq 5$; middle tier, 6 to 7 ; highest tier, $\geq 8$ ) were coded as category variables in additive models applying prior reviews as a guide [47, 48].

2.4.4. Sensitivity Analysis. Aiming to investigate the stability of our meta-analyses' results, we applied two methods in the sensitivity analysis. First, we chose the trim and fill technique to perform a sensitivity analysis in all the reviewed RCTs and in trials that only recruited healthy individuals. Second, the negative control group was chosen over the positive control 
group when the study consisted of two or more control groups. Consequently, we conducted a sensitivity analysis to examine whether involving different control groups would yield different results. The publication bias was appraised for using the funnel plot as well as statistical tests (Egger's test and Begg's test).

The meta-analyses were performed based on at least two trials utilizing software Stata version 12.0 (available from the website for purchasing: https://www.stata.com/products/). A two-sided $P<0.05$ was defined as statistically significant.

\section{Results}

3.1. Study Selection. Figure 1 demonstrates the study selection process. A total of 444 studies were considered for inclusion from four English databases and two Chinese databases. We screened 195 relevant studies based on their abstracts and titles after removing duplicates and then excluded 146 studies because they failed to meet the inclusion criteria. We then reviewed 49 full-text articles and identified a total of 25 RCTs that were eligible for this review [49-73], including 16 English articles and nine Chinese articles. The main reasons for exclusion included studies with duplicated publications, those with a nonrandomized design, and studies that did not contain valuable data. Other main reasons included outcomes with non-self-reported measurements or other self-rated scales except PSQI and those combined Tai Chi exercise with other interventions.

3.2. Characteristics of Included Studies. The principal characteristics of all included RCTs are shown in Table 1. A total of 1858 adults (average age from 50 to 75 years old) were involved in eligible RCTs with 951 individuals assigned to the Tai Chi Chuan intervention group. The included RCTs were conducted between 2004 and 2018 from six countries (11 trials in the U.S, 10 in China, and one each in Japan, Vietnam, Italy, and Iran, respectively). The treatment duration of the included studies ranged from 8 to 36 weeks, and the follow-up time ranged between 8 and 64 weeks. The frequency of intervention varied widely ranging from once per week to 7 times per week, and every class duration was 30 to $120 \mathrm{~min}$. An estimate was then made that the treatment duration was approximately $40.93 \pm 23.22(h$, mean \pm SD), and each class lasted $57.08 \pm 23.60$ (min, mean $\pm \mathrm{SD}$ ). Among all the eligible studies, 10 trials evaluated the effects of Tai Chi Chuan for sleep quality in healthy individuals [49-53, 56, 58, 59, 62-68, 71, 73-76]; four in fibromyalgia patients $[54,57,64,69,72,77,78]$; three in cancer survivors $[61,68,70,79-81]$; two in patients who experienced stroke $[55,60,70,82]$, heart disease $[66,67,83,84]$, and depression $[71,73,85,86]$; and one each in people with chronic kidney disease [65] and arthritis [69]. The Tai Chi Chuan intervention in the majority of included studies was subjected to the Yang style [49, 50, 52, 54-61, 63-73]. Chen style [53] and Tai Chi Ball $[51,62]$ were also adopted in the remaining studies. Notably, the control groups were given diverse interventions such as education, routine daily activity, usual care, waiting list, acupuncture, and some other active exercises. No adverse event was reported in all the included trials.

\section{Methodological Quality}

As illustrated in Table 2, the PEDro scores of the majority $(96.00 \%)$ of the reviewed trials are 6 points or greater, which reflects a moderate to high literature quality. The most common flaws were that participants and therapists in most of the RCTs were unblinded to the intervention, and 14 studies failed to blind assessors $[50,51,53,55,56,58,62-67,70,71]$. Furthermore, the process of concealed allocation was reported in eight studies $[49,52,54,59-61,68,72]$. Meanwhile, the intention-to-treat analysis was rated positive in seven studies $[49,52,54,59,60,68,72]$ and more than $15 \%$ of participants in the five studies dropped out during the intervention $[49,58,68,72,73]$. The remaining items were recorded positive in all the involved studies.

4.1. Meta-Analysis of the Effects of Tai Chi Chuan on Sleep Quality. A total of 25 reviewed studies [49-73] were eligible for the meta-analysis which yielded a moderate effect of Tai Chi Chuan in improving subjective sleep quality in comparison to control interventions $(\mathrm{SMD}=-0.512,95 \% \mathrm{CI}$ $[-0.767,-0.257], P<0.001$; Figure 2). However, a severe statistical heterogeneity was detected $\left(I^{2}=85.8 \%\right.$; $\left.\chi^{2}=168.45, \mathrm{df}=24, P<0.001\right)$.

\subsection{Impact of Tai Chi Chuan on Sleep Quality in Healthy} Adults. Because a substantial amount of heterogeneity was observed, we ran a subgroup analysis on the basis of various conditions of the involved samples (see Figure 2). 10 trials recruited healthy subjects $[49-53,56,58,59,62,63]$. The merged results assumed that Tai Chi Chuan exercise elicited a moderate to large effect in people with good health $(\mathrm{SMD}=-0.684,95 \%$ CI $[-1.056,-0.311], P<0.001)$ in comparison with the control group. However, significant heterogeneity among studies still existed $\left(I^{2}=84.0 \%\right.$, $\chi^{2}=56.18, \mathrm{df}=9, P<0.001 ;$ Figure 2).

4.2.1. Geographic Population. Preplanned subgroup analyses were conducted according to two geographic populations including 422 Asians [51, 53, 56, 58, 62, 63] and 357 Americans [49, 50, 52, 59] (see Figure 3). The subgroup analysis results indicated that the pooled effects on sleep quality for the six studies recruiting the Asian population were large $(\mathrm{SMD}=-0.977,95 \%$ CI $[-1.446,-0.508]$, $P<0.001)$ and had severe statistical heterogeneity $\left(I^{2}=80.7 \%, \chi^{2}=25.96, \mathrm{df}=5, P<0.001\right)$. In contrast, the subgroup of trials that enrolled the American population did not reveal any significant change of sleep quality $(\mathrm{SMD}=-0.259,95 \% \mathrm{CI}[-0.624,0.105], P=0.164)$, and the results were substantially heterogeneous $\left(I^{2}=64.4 \%\right.$, $\left.\chi^{2}=8.43, \mathrm{df}=3, P=0.038\right)$. 


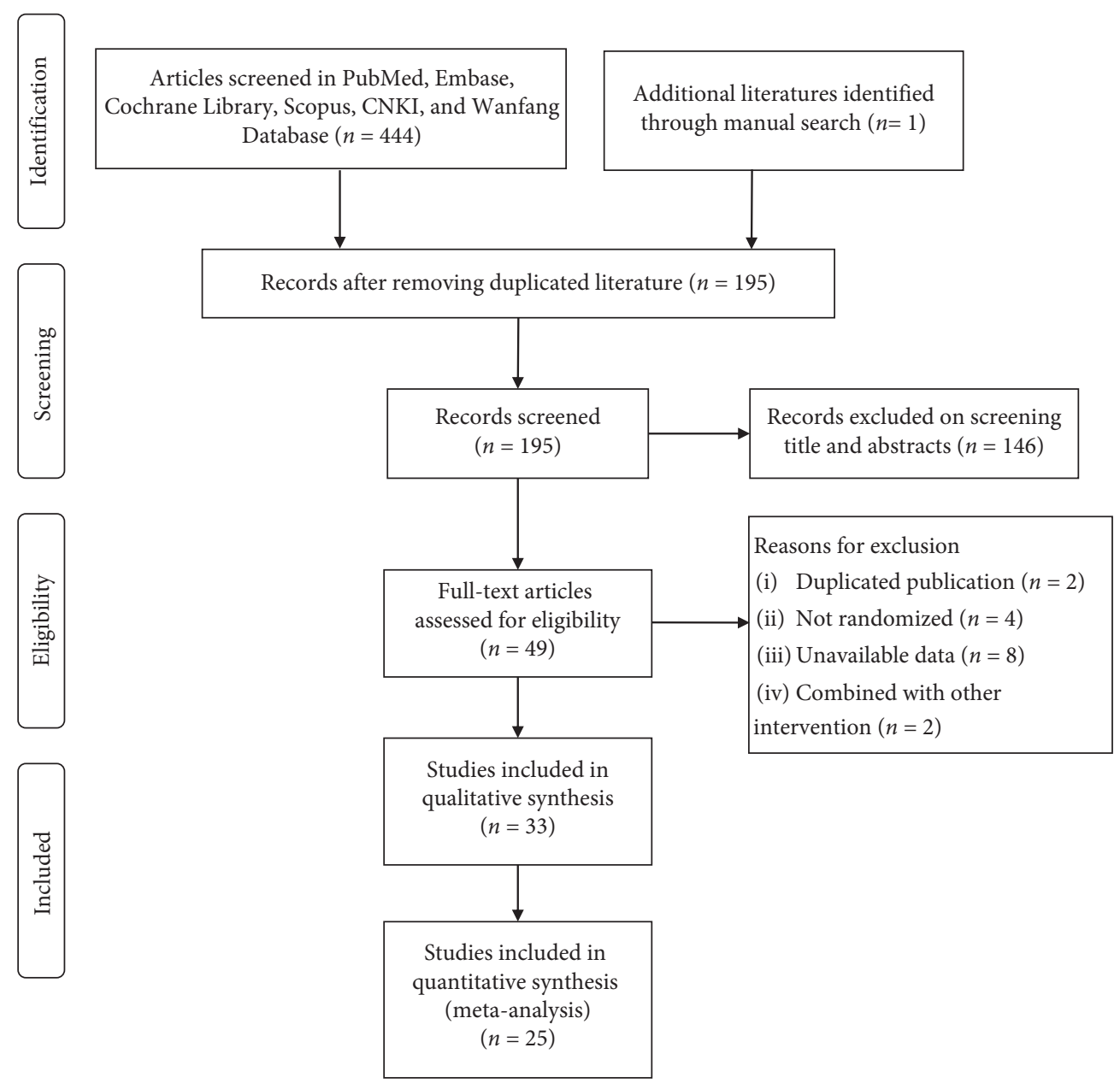

FIGURE 1: PRISMA flow diagram for screening and identifying eligible studies.

4.3. Impact of Tai Chi Chuan on Sleep Quality in the Clinical Population. As illustrated in Figure 4, 15 RCTs targeted the clinical population $[54,55,57,60,61,64-73]$. The pooled results reflected that Tai Chi exercise yielded small effects on sleep quality $(\mathrm{SMD}=-0.395,95 \%$ CI $[-0.742,-0.047]$, $P=0.026)$ and the pooled effect size was notably heterogeneous $\left(I^{2}=86.7 \%, \chi^{2}=105.41, \mathrm{df}=14, P<0.001\right)$. The subgroup analyses of these 15 trials were tailored on the basis of various disease types.

4.3.1. Stroke. Two studies estimated the effects of Tai Chi Chuan on sleep quality in people who have experienced strokes $[55,60]$. In comparison to the control intervention, the aggregated results revealed that Tai Chi Chuan did not significantly improve sleep $(\mathrm{SMD}=0.196 ; 95 \%$ CI $[-0.143$, $0.535]$; $P=0.258$ ). No statistical heterogeneity was present in ESs between studies $\left(I^{2}=0 \%, \chi^{2}=0.64, \mathrm{df}=1, P=0.424\right)$.

4.3.2. Fibromyalgia. Four RCTs [54, 57, 64, 72] recruited fibromyalgia patients, and the pooled results demonstrated that Tai Chi exercise led to a notable advancement in sleep quality, with a small to moderate effect $(\mathrm{SMD}=-0.446 ; 95 \%$ CI $[-0.674,-0.219] ; P<0.001)$. We found no statistical heterogeneity in ESs among the four studies $\left(I^{2}=0 \%\right.$, $\left.\chi^{2}=1.56, \mathrm{df}=3, P=0.668\right)$.

4.3.3. Cancer. Three RCTs tested the impact of Tai Chi Chuan on the sleep quality of cancer survivors [61, 68, 70]. One trial reported that Tai Chi Chuan had a better sleeppromoting impact compared with control interventions [70], but the pooled results did not sustain the positive effects of Tai Chi Chuan in enhancing sleep quality ( $\mathrm{SMD}=-0.041$; 95\% CI $[-1.397,1.315] ; P=0.953)$. The statistical heterogeneity in ESs among studies was extremely severe $\left(I^{2}=95.7 \%, \chi^{2}=46.39, \mathrm{df}=2, P<0.001\right)$.

4.3.4. Depression. Two of the reviewed studies recruited subjects with depression $[71,73]$ and indicated no significant effect on subjective sleep quality (SMD, $-0.875 ; 95 \%$ CI $[-1.760,0.010] ; \quad P=0.053)$ with severe heterogeneity $\left(I^{2}=76.3 \%, \chi^{2}=4.22, \mathrm{df}=1, P=0.040\right)$.

4.3.5. Heart Disease. Two studies enrolled patients with heart disease $[66,67,83,84]$. Among all the subgroup analyses based on diseases, these two trials had the largest pooled effects on sleep disorders $(\mathrm{SMD}=-1.132 ; 95 \% \mathrm{CI}$ 
TABLE 1: Characteristics of included studies.

\begin{tabular}{|c|c|c|c|c|c|c|c|c|}
\hline $\begin{array}{l}\text { Author, } \\
\text { year, } \\
\text { country }\end{array}$ & Primary report & $\begin{array}{l}\text { Sample } \\
\text { size, } \\
\text { mean } \\
\text { age } \\
\text { (years) }\end{array}$ & $\begin{array}{l}\text { Follow- } \\
\text { up } \\
\text { (weeks) }\end{array}$ & $\begin{array}{c}\text { Outcome } \\
\text { measurements }\end{array}$ & $\begin{array}{l}\text { Experimental } \\
\text { group } \\
\text { intervention } \\
(n)\end{array}$ & $\begin{array}{l}\text { Training } \\
\text { program }\end{array}$ & $\begin{array}{l}\text { Control group } \\
\text { intervention }(n)\end{array}$ & $\begin{array}{l}\text { Treatment } \\
\text { program }\end{array}$ \\
\hline $\begin{array}{l}\text { Li et al. } \\
(2004) \text {, } \\
\text { USA }\end{array}$ & Healthy & $\begin{array}{c}118 \\
75.21\end{array}$ & NR & PSQI & $\begin{array}{l}\text { 8-form Yang } \\
\text { style Tai Chi } \\
\quad(n=62)\end{array}$ & $\begin{array}{c}60 \text { min class } \\
\text { program, } 3 \\
\text { times each week } \\
\text { for } 24 \text { weeks }\end{array}$ & $\begin{array}{c}\text { Low-impact } \\
\text { exercise }(n=56)\end{array}$ & $\begin{array}{l}60 \text { min class } \\
\text { program, } 3 \\
\text { times each } \\
\text { week, for } 24 \\
\text { weeks }\end{array}$ \\
\hline $\begin{array}{l}\text { Frye et al. } \\
\text { (2007), } \\
\text { USA }\end{array}$ & Healthy & $84,69.2$ & 14 & PSQI & $\begin{array}{l}\text { 10-form Yang } \\
\text { style Tai Chi } \\
\quad(n=31)\end{array}$ & $\begin{array}{c}60 \text { min class } \\
\text { program, } 3 \\
\text { times each week } \\
\text { for } 12 \text { weeks }\end{array}$ & $\begin{array}{l}\text { No exercise } \\
\quad(n=23)\end{array}$ & NR \\
\hline $\begin{array}{l}\text { Han et al. } \\
\text { (2008), } \\
\text { China }\end{array}$ & Healthy & $\begin{array}{l}\text { 64, NR } \\
\text { (55 years } \\
\text { and } \\
\text { older })\end{array}$ & NR & PSQI & $\begin{array}{l}\text { Tai Chi Ball } \\
\quad(n=32)\end{array}$ & $\begin{array}{c}45 \text { min class } \\
\text { program, } 3 \\
\text { times each week } \\
\text { for } 36 \text { weeks }\end{array}$ & $\begin{array}{c}\text { Aerobic } \\
\text { exercise }(n=32)\end{array}$ & $\begin{array}{l}45 \text { min class } \\
\text { program, } 3 \\
\text { times each week } \\
\text { for } 36 \text { weeks }\end{array}$ \\
\hline $\begin{array}{l}\text { Irwin et al. } \\
\text { (2008), } \\
\text { USA }\end{array}$ & Healthy & $\begin{array}{l}112 \\
60.65\end{array}$ & 25 & PSQI & $\begin{array}{c}\text { Yang style Tai } \\
\text { Chi }(n=59)\end{array}$ & $\begin{array}{l}40 \text { min class } \\
\text { program, } 3 \\
\text { times each week } \\
\text { for } 16 \text { weeks }\end{array}$ & $\begin{array}{l}\text { Health } \\
\text { education } \\
(n=53)\end{array}$ & $\begin{array}{l}40 \text { min class } \\
\text { program, } 3 \\
\text { times each } \\
\text { week, for } 16 \\
\text { weeks }\end{array}$ \\
\hline $\begin{array}{l}\text { Liu et al. } \\
\text { (2010), } \\
\text { China }\end{array}$ & Healthy & $82,66.03$ & NR & PSQI & $\begin{array}{l}\text { Yang style Tai } \\
\text { Chi }(n=43)\end{array}$ & $\begin{array}{l}30 \text { min class } \\
\text { program, } 5 \\
\text { times each week } \\
\text { for } 8 \text { weeks } \\
60 \text { min class }\end{array}$ & $\begin{array}{l}\text { No exercise } \\
\qquad(n=39)\end{array}$ & NR \\
\hline $\begin{array}{l}\text { Wang et al. } \\
\text { (2010), } \\
\text { USA }\end{array}$ & Fibromyalgia & $66,50.10$ & 24 & PSQI & $\begin{array}{l}\text { Classic Yang } \\
\text { style Tai Chi } \\
\quad(n=33)\end{array}$ & $\begin{array}{c}\text { program, } 2 \\
\text { times per week } \\
+20 \text { min home- } \\
\text { based program } \\
\text { every day, for } \\
12 \text { weeks }\end{array}$ & $\begin{array}{l}\text { Wellness } \\
\text { education } \\
(n=33)\end{array}$ & $\begin{array}{l}60 \text { min class } \\
\text { program, } 2 \\
\text { times each } \\
\text { week, for } 12 \\
\text { weeks }\end{array}$ \\
\hline $\begin{array}{l}\text { Wang et al. } \\
(2010) \text {, } \\
\text { Japan }\end{array}$ & Stroke & $\begin{array}{l}\text { 34, NR } \\
\text { (50 years } \\
\text { and } \\
\text { older) }\end{array}$ & NR & PSQI & $\begin{array}{l}\text { Classic Yang } \\
\text { style Tai Chi } \\
\quad(n=17)\end{array}$ & $\begin{array}{l}50 \text { min class } \\
\text { program, } 1 \text { time } \\
\text { each week for } \\
12 \text { weeks }\end{array}$ & $\begin{array}{l}\text { Rehabilitation } \\
\text { program } \\
(n=17)\end{array}$ & $\begin{array}{l}80 \text { min class } \\
\text { program, } 1 \text { time } \\
\text { each week, for } \\
12 \text { weeks }\end{array}$ \\
\hline $\begin{array}{l}\text { Hosseini } \\
\text { et al. } \\
\text { (2011), Iran }\end{array}$ & Healthy & $\begin{array}{l}\text { 62, NR } \\
(65 \text { years } \\
\text { and } \\
\text { older })\end{array}$ & NR & PSQI & $\begin{array}{c}\text { Yang style Tai } \\
\text { Chi }(n=27)\end{array}$ & $\begin{array}{l}5 \text { min at the } \\
\text { first session and } \\
5 \text { min added in } \\
\text { each following } \\
\text { week, } 3 \text { times } \\
\text { each week for } \\
12 \text { weeks }\end{array}$ & $\begin{array}{l}\text { Routine daily } \\
\text { activities } \\
\quad(n=29)\end{array}$ & NR \\
\hline $\begin{array}{l}\text { Jones et al. } \\
(2012) \\
\text { USA }\end{array}$ & Fibromyalgia & $98,54.00$ & NR & PSQI & $\begin{array}{l}\text { 8-form Yang } \\
\text { style Tai Chi } \\
\quad(n=51)\end{array}$ & $\begin{array}{l}90 \text { min class } \\
\text { program, } 2 \\
\text { times each week } \\
\text { for } 12 \text { weeks }\end{array}$ & $\begin{array}{c}\text { Education } \\
\text { intervention } \\
(n=47)\end{array}$ & $\begin{array}{l}90 \text { min class } \\
\text { program, } 2 \\
\text { times each } \\
\text { week, for } 12 \\
\text { weeks }\end{array}$ \\
\hline $\begin{array}{l}\text { Nguyen } \\
\text { et al. } \\
\text { (2012), } \\
\text { Vietnam }\end{array}$ & Healthy & $96,68.90$ & NR & PSQI & $\begin{array}{c}\text { 24-form Yang } \\
\text { style Tai Chi } \\
\quad(n=48)\end{array}$ & $\begin{array}{c}60 \text { min class } \\
\text { program, } 2 \\
\text { times each week } \\
\text { for } 24 \text { weeks } \\
120 \text { min class }\end{array}$ & $\begin{array}{l}\text { Routine daily } \\
\text { activities } \\
(n=48)\end{array}$ & NR \\
\hline $\begin{array}{l}\text { Irwin et al. } \\
(2014) \\
\text { USA }\end{array}$ & Healthy & $98,65.33$ & 64 & PSQI & $\begin{array}{c}\text { Yang style Tai } \\
\text { Chi }(n=48)\end{array}$ & $\begin{array}{c}120 \text { min class } \\
\text { and home- } \\
\text { based program, } \\
1 \text { time each } \\
\text { week for } 16 \\
\text { weeks }\end{array}$ & $\begin{array}{l}\text { Sleep seminar } \\
\text { education } \\
\quad(n=50)\end{array}$ & $\begin{array}{l}120 \text { min class } \\
\text { program, } 1 \text { time } \\
\text { each week, for } \\
16 \text { weeks }\end{array}$ \\
\hline
\end{tabular}


TABle 1: Continued.

\begin{tabular}{|c|c|c|c|c|c|c|c|c|}
\hline $\begin{array}{l}\text { Author, } \\
\text { year, } \\
\text { country }\end{array}$ & Primary report & $\begin{array}{l}\text { Sample } \\
\text { size, } \\
\text { mean } \\
\text { age } \\
\text { (years) }\end{array}$ & $\begin{array}{l}\text { Follow- } \\
\text { up } \\
\text { (weeks) }\end{array}$ & $\begin{array}{c}\text { Outcome } \\
\text { measurements }\end{array}$ & $\begin{array}{l}\text { Experimental } \\
\text { group } \\
\text { intervention } \\
(n)\end{array}$ & $\begin{array}{l}\text { Training } \\
\text { program }\end{array}$ & $\begin{array}{l}\text { Control group } \\
\text { intervention }(n)\end{array}$ & $\begin{array}{l}\text { Treatment } \\
\text { program }\end{array}$ \\
\hline $\begin{array}{l}\text { Taylor- } \\
\text { Piliae et al. } \\
\text { (2014), } \\
\text { USA }\end{array}$ & Stroke & $97,69.90$ & NR & PSQI & $\begin{array}{l}\text { Tai Chi easy } \\
\quad(n=53)\end{array}$ & $\begin{array}{c}60 \text { min class } \\
\text { program, } 3 \\
\text { times each week } \\
\text { for } 12 \text { weeks }\end{array}$ & $\begin{array}{l}\text { Usual care } \\
(n=48)\end{array}$ & $\begin{array}{l}\text { Weekly phone } \\
\text { calls, for } 12 \\
\text { weeks }\end{array}$ \\
\hline $\begin{array}{l}\text { Larkey } \\
\text { et al. } \\
\text { (2015), } \\
\text { USA }\end{array}$ & Breast cancer & $87,58.80$ & 12 & PSQI & $\begin{array}{l}\text { Yang style Tai } \\
\text { Chi }(n=42)\end{array}$ & $\begin{array}{c}30 \text { min home- } \\
\text { based program, } \\
5 \text { times each } \\
\text { week for } 12 \\
\text { weeks }\end{array}$ & $\begin{array}{l}\text { Sham Qigong } \\
\qquad(n=45)\end{array}$ & $\begin{array}{c}30 \text { min home- } \\
\text { based program, } \\
5 \text { times each } \\
\text { week, for } 12 \\
\text { weeks }\end{array}$ \\
\hline $\begin{array}{l}\text { Liu et al. } \\
\text { (2015), } \\
\text { China }\end{array}$ & Healthy & $84,63.30$ & NR & PSQI & $\begin{array}{l}\text { Tai Chi Ball } \\
\quad(n=44)\end{array}$ & $\begin{array}{c}60 \text { min class } \\
\text { program, } 3 \\
\text { times each week } \\
\text { for } 18 \text { weeks }\end{array}$ & $\begin{array}{l}\text { No exercise } \\
\quad(n=40)\end{array}$ & NR \\
\hline $\begin{array}{l}\text { Yuan et al. } \\
\text { (2016), } \\
\text { China }\end{array}$ & $\begin{array}{l}\text { Heart failure } \\
\text { combined with } \\
\text { depression }\end{array}$ & $60,66.90$ & NR & PSQI & $\begin{array}{c}\text { 24-form Yang } \\
\text { style Tai Chi } \\
\quad(n=32)\end{array}$ & $\begin{array}{c}30 \text { min class } \\
\text { program, } 5 \\
\text { times each week } \\
\text { for } 12 \text { weeks }\end{array}$ & $\begin{array}{l}\text { Usual care } \\
(n=30)\end{array}$ & NR \\
\hline $\begin{array}{l}\text { Liu et al. } \\
\text { (2016), } \\
\text { China }\end{array}$ & Healthy & $63,66.05$ & 8 & PSQI & $\begin{array}{c}\text { 24-form Yang } \\
\text { style Tai Chi } \\
\quad(n=32)\end{array}$ & $\begin{array}{c}60 \text { min class } \\
\text { program, } 5 \\
\text { times each week } \\
\text { for } 16 \text { weeks }\end{array}$ & $\begin{array}{c}\text { No exercise } \\
\quad(n=31)\end{array}$ & NR \\
\hline $\begin{array}{l}\text { Bongi et al. } \\
\text { (2016), } \\
\text { Italy }\end{array}$ & Fibromyalgia & $44,52.24$ & NR & PSQI & $\begin{array}{l}\text { Unreported } \\
\text { style Tai Chi } \\
\quad(n=22)\end{array}$ & $\begin{array}{c}60 \text { min class } \\
\text { program, } 2 \\
\text { times each week } \\
\text { for } 16 \text { weeks }\end{array}$ & $\begin{array}{c}\text { Educational } \\
\text { Session }(n=22)\end{array}$ & No session \\
\hline $\begin{array}{l}\text { Fan et al. } \\
(2016) \\
\text { China }\end{array}$ & $\begin{array}{l}\text { Hemodialysis } \\
\text { patients }\end{array}$ & $71,51.23$ & NR & PSQI & $\begin{array}{c}\text { 24-form Yang } \\
\text { style Tai Chi } \\
\quad(n=34)\end{array}$ & $\begin{array}{l}20 \text { min class } \\
\text { program, } 2 \\
\text { times each week } \\
\text { for the first } \\
12 \text { weeks and } \\
45 \text { min home- } \\
\text { based program, } \\
3 \text { times each } \\
\text { week for the } \\
\text { next } 12 \text { weeks }\end{array}$ & $\begin{array}{l}\text { Usual care } \\
(n=37)\end{array}$ & NR \\
\hline $\begin{array}{l}\text { Chen et al. } \\
\text { (2017), } \\
\text { China }\end{array}$ & $\begin{array}{l}\text { Coronary heart } \\
\text { disease }\end{array}$ & $\begin{array}{l}115 \\
60.87\end{array}$ & NR & PSQI & $\begin{array}{c}\text { 24-form Yang } \\
\text { style Tai Chi } \\
\quad(n=57)\end{array}$ & $\begin{array}{c}60 \text { min class } \\
\text { program, } 3 \\
\text { times each week } \\
\text { for } 12 \text { weeks }\end{array}$ & $\begin{array}{l}\text { Usual care } \\
(n=58)\end{array}$ & NR \\
\hline $\begin{array}{l}\text { Irwin et al. } \\
\text { (2017), } \\
\text { USA }\end{array}$ & Breast cancer & $90,59.84$ & 60 & PSQI, AISI & $\begin{array}{l}\text { Yang style Tai } \\
\text { Chi }(n=45)\end{array}$ & $\begin{array}{l}120 \text { min class } \\
\text { program, } 1 \text { time } \\
\text { each week for } \\
12 \text { weeks }\end{array}$ & $\begin{array}{c}\text { Cognitive } \\
\text { behavioral } \\
\text { therapy }(n=45)\end{array}$ & $\begin{array}{l}120 \text { min class } \\
\text { program, } 1 \text { time } \\
\text { each week for } \\
12 \text { weeks }\end{array}$ \\
\hline $\begin{array}{l}\text { Lv et al. } \\
(2017) \\
\text { China }\end{array}$ & Arthritis & $46,64.57$ & NR & PSQI & $\begin{array}{l}\text { 8-form Yang } \\
\text { style Tai Chi } \\
\quad(n=23)\end{array}$ & $\begin{array}{c}60 \text { min class } \\
\text { program, } 3 \\
\text { times each week } \\
\text { for } 24 \text { weeks }\end{array}$ & $\begin{array}{l}\text { Wellness } \\
\text { education } \\
(n=23)\end{array}$ & $\begin{array}{l}60 \text { min class } \\
\text { program, } 2 \\
\text { times each week } \\
\text { for } 24 \text { weeks }\end{array}$ \\
\hline $\begin{array}{l}\text { McQuade } \\
\text { et al. } \\
\text { (2017), } \\
\text { USA }\end{array}$ & Prostate cancer & $52,63.60$ & 12 & $\begin{array}{l}\text { PSQI, } 18 \text {-item } \\
\text { self-rated } \\
\text { questionnaire }\end{array}$ & $\begin{array}{l}8 \text {-form Yang } \\
\text { style Tai Chi } \\
\quad(n=26)\end{array}$ & $\begin{array}{l}40 \text { min class } \\
\text { program, } 1 \text { time } \\
\text { each week for } 6 \\
\text { to } 8 \text { weeks }\end{array}$ & $\begin{array}{l}\text { Wait list } \\
(n=24)\end{array}$ & NR \\
\hline $\begin{array}{l}\text { Rao et al. } \\
(2018) \\
\text { China }\end{array}$ & Depression & $60,54.62$ & NR & PSQI & $\begin{array}{c}\text { 24-form Yang } \\
\text { style Tai Chi } \\
\quad(n=30)\end{array}$ & $\begin{array}{l}60 \text { min class } \\
\text { program, } 5 \\
\text { times each week } \\
\text { for } 8 \text { weeks }\end{array}$ & $\begin{array}{l}\text { Acupuncture } \\
\quad(n=30)\end{array}$ & $\begin{array}{c}30 \mathrm{~min} \\
\text { treatment, } 5 \\
\text { times each week } \\
\text { for } 8 \text { weeks }\end{array}$ \\
\hline
\end{tabular}


TABLE 1: Continued.

\begin{tabular}{|c|c|c|c|c|c|c|c|c|}
\hline $\begin{array}{l}\text { Author, } \\
\text { year, } \\
\text { country }\end{array}$ & Primary report & $\begin{array}{l}\text { Sample } \\
\text { size, } \\
\text { mean } \\
\text { age } \\
\text { (years) }\end{array}$ & $\begin{array}{l}\text { Follow- } \\
\text { up } \\
\text { (weeks) }\end{array}$ & $\begin{array}{c}\text { Outcome } \\
\text { measurements }\end{array}$ & $\begin{array}{l}\text { Experimental } \\
\text { group } \\
\text { intervention } \\
(n)\end{array}$ & $\begin{array}{l}\text { Training } \\
\text { program }\end{array}$ & $\begin{array}{l}\text { Control group } \\
\text { intervention }(n)\end{array}$ & $\begin{array}{l}\text { Treatment } \\
\text { program }\end{array}$ \\
\hline $\begin{array}{l}\text { Wang et al. } \\
\text { (2018), } \\
\text { USA }\end{array}$ & Fibromyalgia & $\begin{array}{c}111, \\
52.10\end{array}$ & 52 & PSQI & $\begin{array}{l}\text { Classic Yang } \\
\text { style Tai Chi } \\
\quad(n=36)\end{array}$ & $\begin{array}{l}60 \text { min class } \\
\text { program, } 2 \\
\text { times each week } \\
\text { for } 24 \text { weeks } \\
60 \text { min class }\end{array}$ & $\begin{array}{c}\text { Aerobic } \\
\text { exercise }(n=75)\end{array}$ & $\begin{array}{c}60 \text { min class } \\
\text { program, } 2 \\
\text { times each week } \\
\text { for } 24 \text { weeks }\end{array}$ \\
\hline $\begin{array}{l}\text { Zhu et al. } \\
\text { (2018), } \\
\text { China }\end{array}$ & Depression & $80, \mathrm{NR}$ & NR & PSQI & $\begin{array}{l}\text { 24-form Yang } \\
\text { style Tai Chi } \\
\quad(n=42)\end{array}$ & $\begin{array}{l}\text { program, } 5 \\
\text { times each week } \\
\text { for the first } 12 \\
\text { weeks and } \\
60 \text { min class } \\
\text { program, } 3 \\
\text { times each week } \\
\text { for the next } 12 \\
\text { weeks }\end{array}$ & $\begin{array}{l}\text { Setting up } \\
\text { exercises to } \\
\text { radio music } \\
\quad(n=38)\end{array}$ & $\begin{array}{l}4 \text { min and } 45 \mathrm{~s} \\
\text { class program, } 5 \\
\text { times each week } \\
\text { for the first } 12 \\
\text { weeks and } 3 \\
\text { times each week } \\
\text { for the next } 12 \\
\text { weeks }\end{array}$ \\
\hline
\end{tabular}

PSQI, Pittsburgh Sleep Quality Index; NR, no report; AISI, Athens Insomnia Severity Index.

$[-1.455,-0.809] ; P<0.001)$ and contained no statistically detected heterogeneity $\left(I^{2}=0 \%, \chi^{2}=0.11, \mathrm{df}=1, P<0.001\right)$.

4.4. Meta-Regression and Additive Models. As shown in Table 3, the results revealed that the methodological quality of included studies significantly correlated with the effects of Tai Chi Chuan for sleep quality, and it was the most important source of heterogeneity with a $P$ value of 0.012 . In addition, geographic population $(P=0.038)$ and training intensity $(P=0.026)$ were also notable factors affecting sleep quality response to Tai Chi Chuan training. Generally, these three covariables (methodological quality, geographic population, and training intensity) could explain the $28.96 \%$, $15.09 \%$, and $17.22 \%$ of the heterogeneity source in sleep quality response to Tai Chi Chuan training, respectively.

Notably, it has been observed that Tai Chi Chuan elicited greater sleep quality improvements in the Asian population $(P<0.001)$ than in the American population $(P=0.226)$. It has also yielded more improvements among subjects who engaged in Tai Chi exercise of low $(P<0.002)$ or moderate $(P<0.001$ training intensity than vigorous intensity $(P<0.579)$. Furthermore, sleep quality enhancements were more significant among studies that belonged in the middle tier $(P<0.001)$ of methodological quality assessed by the PEDro scale than those in the highest tier $(P=0.839)$. The additive statistical models illustrated that after controlling the factors of geographic population and methodological quality of studies, participants could achieve the most considerable sleep-promoting benefits when Tai Chi Chuan was practiced between 60 and $90 \mathrm{~min}$ per session (SMD, -0.795 ; $95 \%$ CI $[-1.246,-0.344]$; $P=0.001)$.

4.5. Publication Bias. A funnel plot was completed based on 25 RCTs [49-73]. According to the visual assessment, no significant evidence of publication bias was identified. This is due to the fact that the spots were roughly symmetric, although several studies seemed to be remarkable outliers (see Figure 5). The results of Begg's test $(P=0.183)$ and Egger's test $(P=0.660)$ also noted the consistency and registered no significant publication bias in the metaanalysis results.

4.6. Sensitivity Analysis. Severe heterogeneity was detected among studies, which indicated that it was necessary to conduct the sensitivity analysis. Firstly, four out of the 25 reviewed studies $[50,59,60,70]$ had two control groups, and the negative control group was chosen over the positive control group in the preplanned meta-analyses. As a result of this, we directed a sensitivity analysis to determine if selecting substituted control groups would yield varying outcomes. A similar result of the sensitivity analysis $(\mathrm{SMD}=-0.497,95 \%$ CI $[-0.773,-0.222], P<0.001)$ was found in comparison to the preplanned meta-analysis $(\mathrm{SMD}=-0.512,95 \%$ CI $[-0.767,-0.257], P<0.001)$. Secondly, the trim and fill analysis was applied in a randomeffects model among all the 25 included trials [49-73], and this was also applied in the 10 trials $[49-53,56,58,59,62,63]$ that solely recruited healthy subjects. The results of the trim and fill technique demonstrated that no trial had been trimmed or filled in both study selection methods, which suggests good stability of the meta-analysis results.

\section{Discussion}

The current review investigated the evidence of the effects of Tai Chi exercise on subjective sleep quality in adults. We conducted the meta-analysis utilizing the extracted data from 25 RCTs with moderate to high quality. The central findings of all the meta-analyses are that (1) Tai Chi Chuan training can yield a moderate effect in improving sleep quality in both healthy and clinical populations; (2) Tai Chi exercise is able to elicit greater sleep-promoting benefits in the healthy population than in the clinical population and 


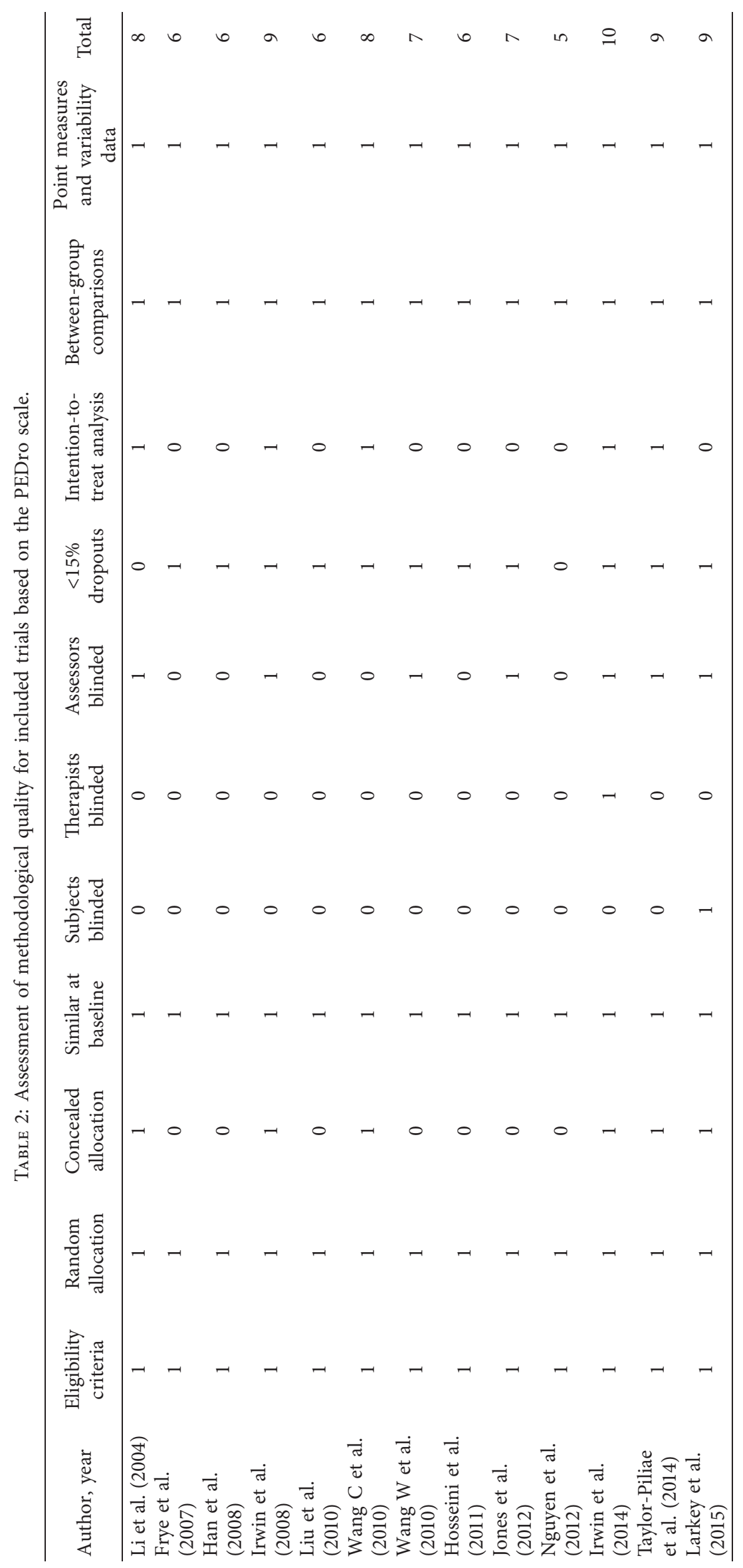




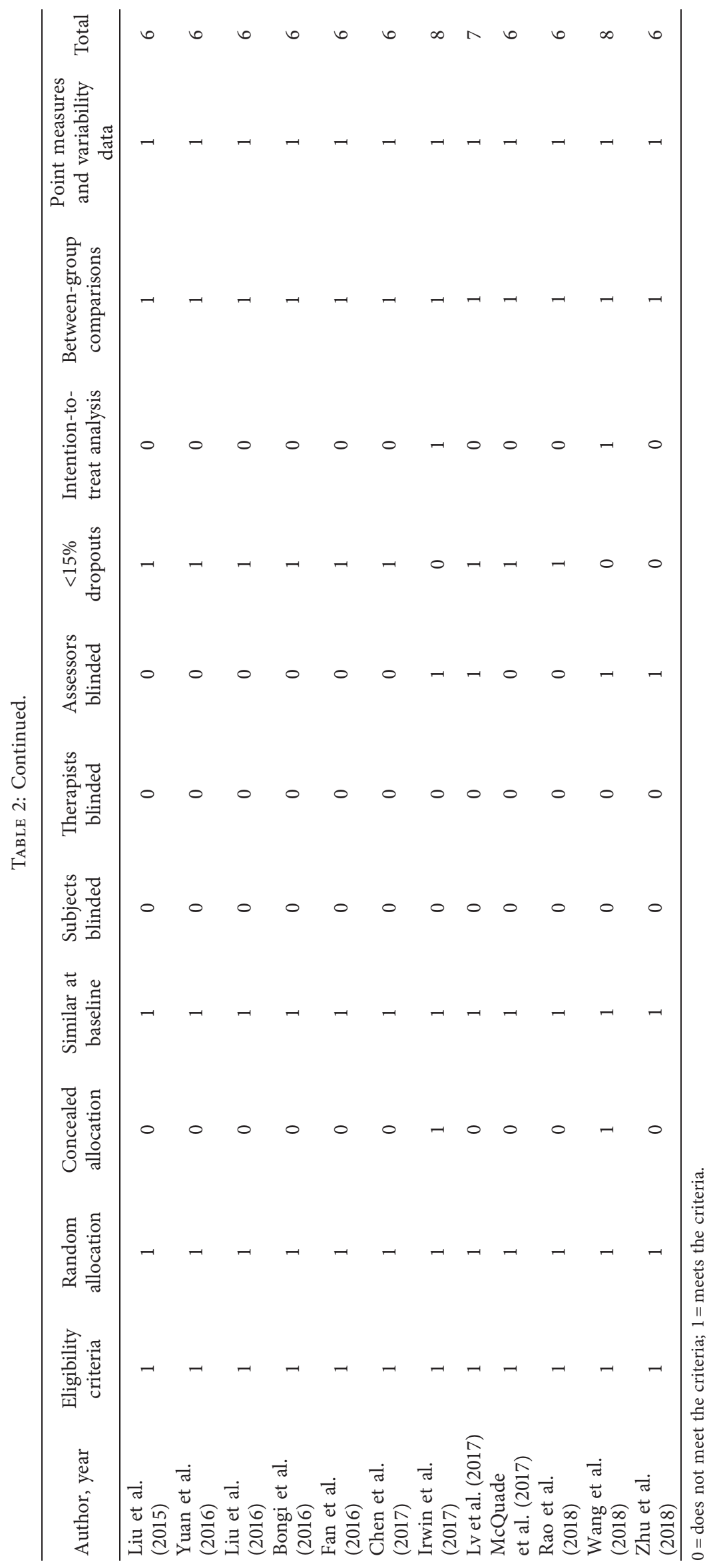




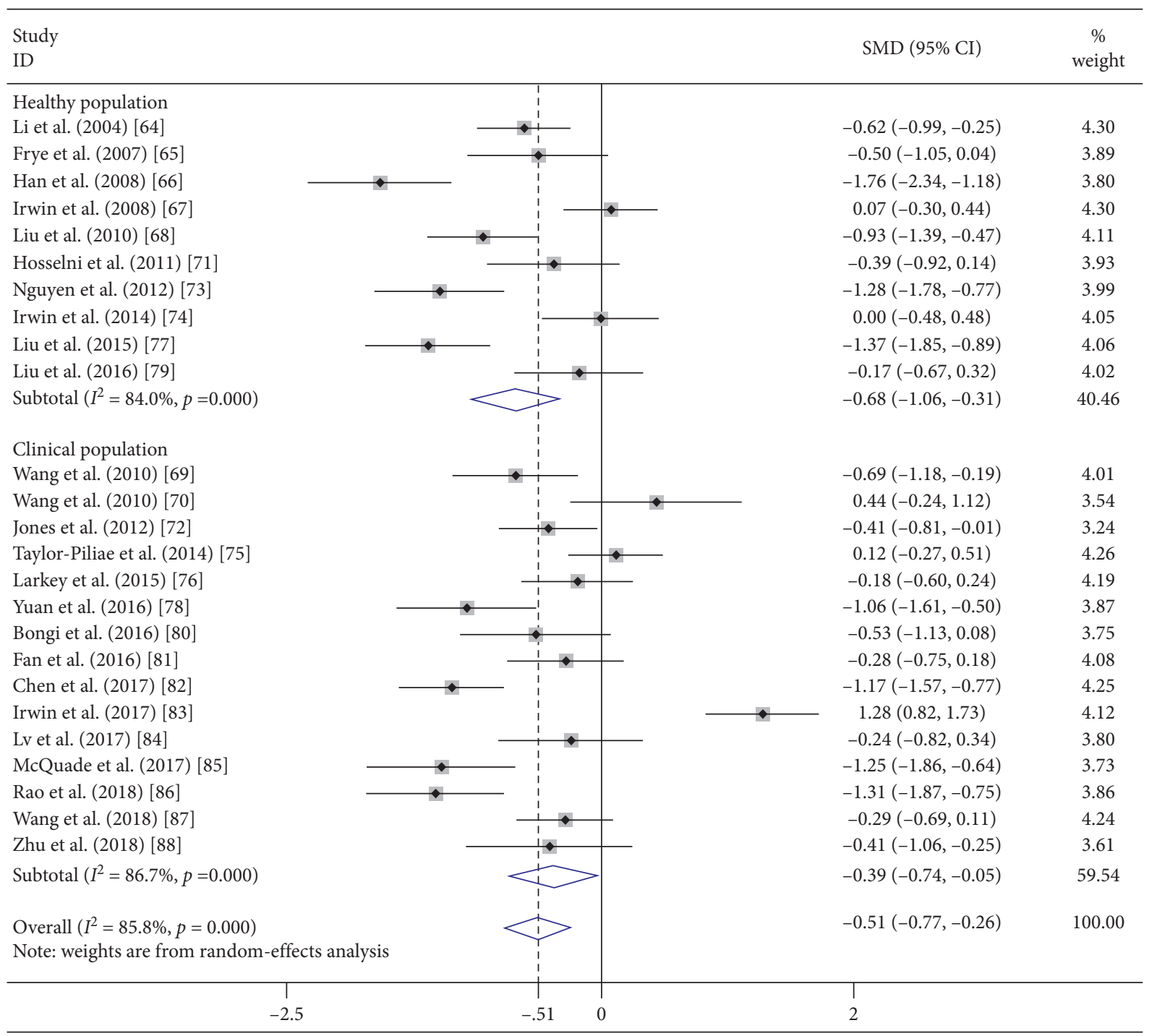

FIGURE 2: Effect of population condition (healthy and clinical) on subjective sleep quality.

more benefits among Asians than in Americans; (3) after controlling methodological quality and geographic population, the optimal Tai Chi training intensity for the Asian population is identified to be between 60 and $90 \mathrm{~min}$ per session.

We generally detected that there was a moderate effect $(\mathrm{SMD}=-0.512,95 \%$ CI $[-0.767,-0.257], P<0.001, k=25)$ of Tai Chi Chuan on subjective sleep quality in all the included samples. Three previous systematic reviews and meta-analyses also evaluated the impact of Tai Chi Chuan training on sleep quality (healthy and clinical populations were pooled together). Our results were more conservative than those reported by $\mathrm{Wu}$ et al. [29] (SMD $=-0.64,95 \% \mathrm{CI}$ $[-0.97,-0.30], P<0.01, k=9)$ and Raman et al. [33] $(\mathrm{SMD}=-0.89,95 \% \mathrm{CI}[-1.50,-0.28], P<0.001, k=8)$ and more progressive than those in the study of Wang et al. [32] $(\mathrm{SMD}=-0.35,95 \%$ CI $[-0.63,-0.07], P=0.016, k=12)$. There appeared to be a lack of consistency between current and previous results, but it should be noted that our findings may be more comprehensive to their results for two main reasons: (a) the number of eligible trials in these three systematic reviews and meta-analyses seemed insufficient, and no study published in Chinese was involved in the reviews of Wang et al. [32] and Raman et al. [33]. On the contrary, we compiled and meta-analyzed more updated RCTs published in both English $(k=16)$ and Chinese $(k=9)$ which implies a more considerable amount of evidence. (b) $\mathrm{Wu}$ et al. [29] included two trials that were published in Chinese in their review. However, the two trials combined Tai Chi Chuan with other interventions (e.g., music therapy), which might have resulted in an overestimation of the aggravated ESs [74, 82]. Therefore, our results may have yielded a more precise representation of the effects of Tai Chi Chuan on sleep quality in both healthy and clinical populations.

Furthermore, we also found that there was a moderate to large effect in nonclinical population $(\mathrm{SMD}=-0.684,95 \%$ CI $[-1.056,-0.311], P<0.001, k=10)$ and a small effect among the clinical population $(\mathrm{SMD}=-0.395,95 \% \mathrm{CI}$ $[-0.742,-0.047], P=0.026, k=15)$. To our knowledge, 


\begin{tabular}{l}
\hline Study \\
ID \\
\hline American \\
Li et al. (2004) [64] \\
Frye et al. (2007) [65] \\
Irwin et al. (2008) [67] \\
Irwin et al. (2014) [74] \\
Subtotal ( $I^{2}=64.4 \%, p=0.038$ ) \\
Asian \\
Han et al. (2008) [66] \\
Liu et al. (2010) [68]
\end{tabular}

FIGURE 3: Effect of geographic population (Americans and Asians) on sleep quality in healthy adults.

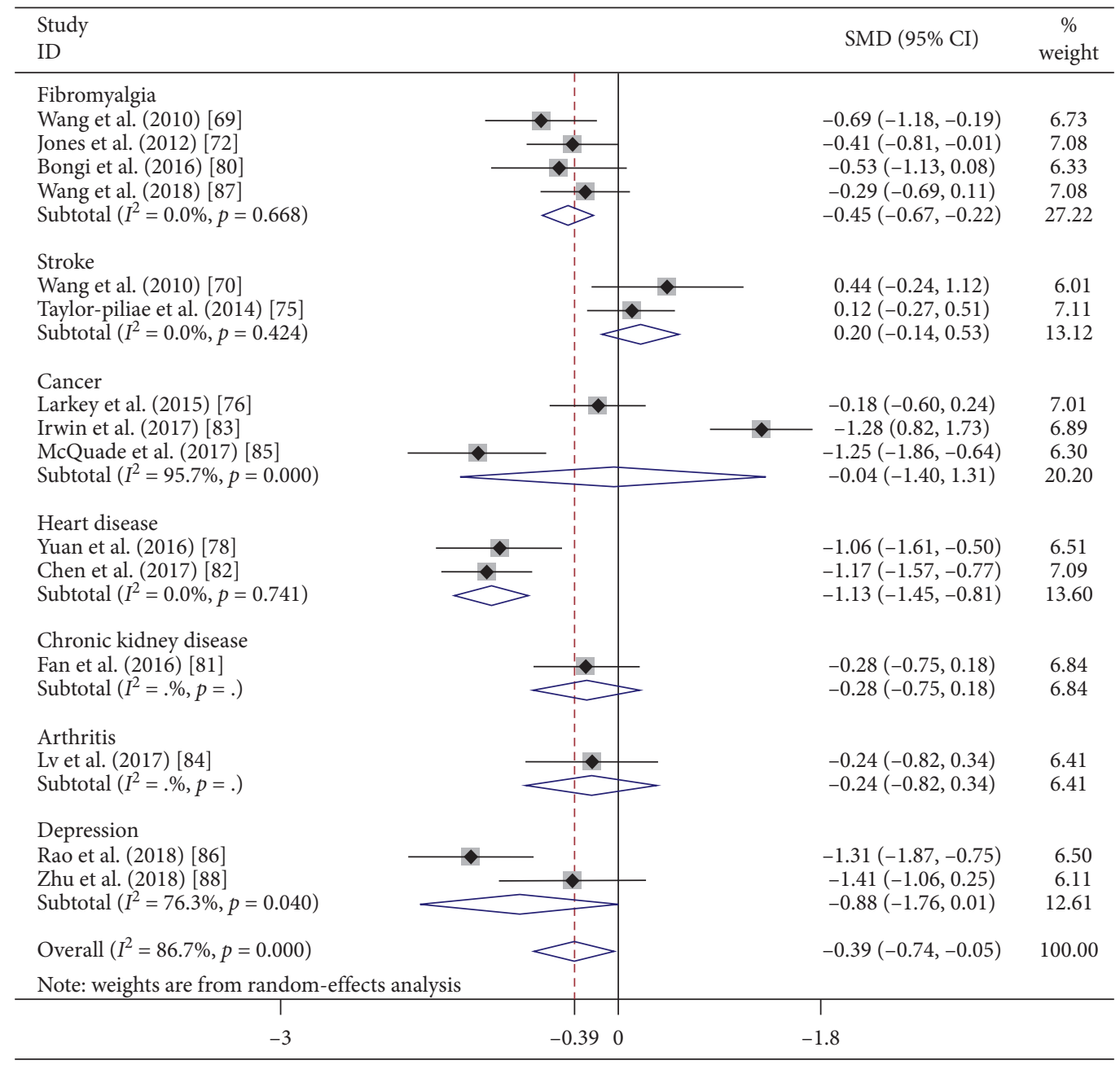

FIGURE 4: Effect of disease type on sleep quality. 
TABLE 3: Meta-regression and additive models: sleep quality change response to Tai Chi exercise.

\begin{tabular}{|c|c|c|c|c|c|}
\hline Moderator category/level & $\beta$ & Adjusted $R^{2}(\%)$ & $P^{1}$ value & Effect size $(95 \% \mathrm{CI})$ & $P^{2}$ value \\
\hline Geographic population & -0.563 & 15.09 & 0.038 & & \\
\hline American $(k=11)$ & & & & $-0.213(-0.558,0.132)$ & 0.226 \\
\hline Asian $(k=13)$ & & & & $-0.778(-1.097,-0.460)$ & $<0.001$ \\
\hline European $(k=1)$ & & & & $-0.527(-1.128,0.075)$ & 0.086 \\
\hline Methodological quality (score) & -0.675 & 28.96 & 0.012 & & \\
\hline Lowest tier, $\leq 5(k=1)$ & & & & $-1.276(-1.781,-0.770)$ & $<0.001$ \\
\hline Middle tier, 6 to $7(k=16)$ & & & & $-0.718(-0.983,-0.453)$ & $<0.001$ \\
\hline Highest tier, $\geq 8(k=8)$ & & & & $-0.041(-0.441,0.385)$ & 0.839 \\
\hline Training intensity (min/session) & -0.930 & 17.22 & 0.026 & & \\
\hline Low, $30 \leq$ time $<60 \quad(k=10)$ & & & & $-0.596(-0.975,-0.218)$ & 0.002 \\
\hline Moderate, $60 \leq$ time $<90(k=12)$ & & & & $-0.647(-0.946,-0.348)$ & $<0.001$ \\
\hline Vigorous, $90 \leq$ time $<120(k=3)$ & & & & $0.286(-0.726,1.298)$ & 0.579 \\
\hline \multicolumn{6}{|l|}{ Additive models: } \\
\hline \multicolumn{6}{|c|}{ (1) Among Asian population; (2) among studies with methodological quality of middle tier (PEDro score: 6 to 7 points). } \\
\hline Low intensity: $30 \leq$ time $<60(\mathrm{~min} /$ session $)$ & & & & $-0.674(-1.215,-0.133)$ & 0.015 \\
\hline Moderate intensity: $60 \leq$ time $<90$ ( $\mathrm{min} /$ session) & & & & $-0.795(-1.246,-0.344)$ & 0.001 \\
\hline
\end{tabular}

$\beta$, regression coefficient; $k$, number of trials; adjusted $R^{2}$, the proportion of the source of heterogeneity explained by each moderator; $P^{1}$ value, the statistical significance of each moderator in the meta-regression model; $P^{2}$ value, the statistical significance of subgroup analyses; effect sizes are generated by subgroup analyses based on the three moderators, respectively.

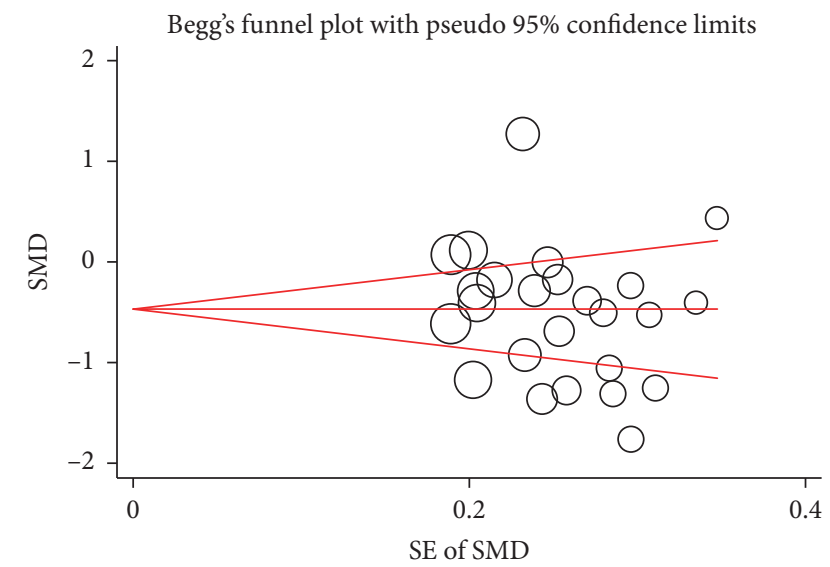

Figure 5: Funnel plot for publication bias. SMD: standardized mean difference; SE: standard error.

however, no systematic review and meta-analysis which separately evaluates the sleep-promoting effects of Tai Chi exercise in healthy and clinical populations has been conducted. Consequently, we can only compare the current results with the previous study provided by our research group at the Nanjing University of Chinese Medicine [34]. The previous review meta-analyzed five eligible RCTs that recruited healthy older adults, and the results indicated that Tai Chi Chuan elicited significant sleep improvements in older people (SMD $=-0.87,95 \%$ CI $[-1.25,-0.49], P<0.01$, $k=5$ ), which were more progressive than our current findings. The differences between the previous and current results are most likely because only five RCTs were included in the previous study (all included in this review) which may have resulted in a lack of accuracy and representation. In addition, Yang et al. [79] conducted a systematic review and meta-analysis of five RCTs to evaluate the impact of exercise (moderate-intensity aerobic exercise or high-intensity resistance exercise) for self-reported sleep quality in healthy adults (288 participants) and found a nearly moderate effect size of 0.47 (95\% CI [0.08, 0.86], $k=5$ ), which was smaller than what was reported by us for Tai Chi Chuan. Unfortunately, we were unable to make any comparison with the previous results in relation to the aggregated effects of Tai Chi exercise on sleep quality among the clinical population as we did not identify any qualified review.

The eligible RCTs in our review enrolled subjects with various physical conditions, including good health, stroke, fibromyalgia, heart disease, cancer, arthritis, depression, and chronic kidney disease. Therefore, one subgroup analysis was administrated based on various physical conditions, the results of which indicated that Tai Chi exercise was more effective in improving sleep quality among healthy adults $(k=10)$ as well as patients with fibromyalgia $(k=4)$ and heart disease $(k=2)$. Nonetheless, only moderate evidence of the impact of Tai Chi exercise on sleep promotion among healthy adults could be preliminarily proved since the results of fibromyalgia and heart disease were yielded by fewer involved trials. Moreover, the other subgroup analysis was to compare the various sleep responses to Tai Chi training between healthy Asian and American populations. The pooled results revealed a demonstrable difference between the two geographic populations that Asian subjects gained statistically significant sleep quality benefits from Tai Chi Chuan, while the Americans did not. One potential cause that may explain the results is as follows: as is known to all, Tai Chi Chuan originates from ancient China and has been developed by the various generations of Chinese people [33]. Simply put, Tai Chi Chuan is fundamentally a tailor-made form of exercise and lifestyle therapy for the body constitutions of Chinese people, which resembles populations from other Asian countries (e.g., Korea and Vietnam) rather than the American population.

Tai Chi exercise was proved efficacious in mitigating poor sleep quality in numerous previous studies [29-32, 34], but its training intensity, frequency, duration, and styles 
varied immensely. Consequently, a particular objective of this review was to identify the combination of Tai Chi Chuan elements that yields the most effective enhancement of sleep quality by utilizing additive models. After controlling the methodological quality of studies, the most practical and clinically relevant finding in this review was that Tai Chi exercise could yield the most significant sleep quality improvement when it was practiced between 60 and $90 \mathrm{~min}$ per session among Asian participants. Several previous systematic reviews recommended the prescriptions of other Tai Chi Chuan elements (style, treatment duration, and frequency) in chronic conditions [18, 26, 75], but no review found any better option of the training intensity (session time) so far. Consequently, our findings might be novel and encouraging with respect to the use of Tai Chi Chuan for chronic conditions.

5.1. How Tai Chi Chuan Elicits Sleep Quality Improvements. Although a number of studies have supported the application of Tai Chi Chuan on adults with poor sleep quality [29-34], the exact biological mechanism remains unclear. It appears that Tai Chi Chuan can enhance the mind-body connection and control through integrating physical, emotional, psychosocial, spiritual, and behavioral elements. The multiple components of Tai Chi Chuan may act through intermediate pathways (e.g., neuroendocrine, immune function, and neurochemical) to improve psychological and behavioral factors associated with poor sleep quality, such as anxiety, depression, and chronic pain [52, 76, 83]. In addition, Montgomery and Dennis [77] claimed that Tai Chi practitioners had higher energy expenditure during the day; thus, they required greater rest at night. Nevertheless, Irwin et al. [52] argued that there were no significant changes in the physical energy expenditure yielded by Tai Chi Chuan, and the sleep enhancement seemed to be independent of energy expenditure. Chao et al. [87] found that the average exercise intensity of Tai Chi Chuan was 3.1 MET (metabolic equivalent). Therefore, Tai Chi Chuan is a low-intensity exercise. As shown in the studies reviewed here, we believe that the gentle movements, slow rhythm, deep diaphragmatic breathing, and emotional relaxation of Tai Chi Chuan can improve the feelings of well-being and ameliorate mental state, which may be the critical factors to the sleep quality improvement. However, the specific mechanism by which Tai Chi Chuan improves sleep quality and other outcomes in the context of these various health conditions are needed to be further studied.

5.2. Tai Chi Chuan and Cognitive Behavioral Therapy. Our findings support the perspective of applying Tai Chi Chuan as a complementary and alternative therapy for poor sleep quality to some degree because the ESs of the changing global PSQI scores elicited by Tai Chi Chuan are approximately equivalent to the ESs of those reported for cognitive behavioral therapy (CBT). Notably, CBT is currently highly recommended as the first-line treatment by both the American College of Physicians as well as the European Sleep Research Society in the management of insomnia
$[35,36,80,84]$. Because CBT has been proven effective for insomnia and is currently widely used, the following question is: why we still need Tai Chi Chuan to treat people with poor sleep quality? First, CBT is an intensive form of intervention that requires proper administration and supervision by highly trained clinicians [88]. Thus, the CBT medical resources may be low cost-efficient and nonpragmatic in usual medical institutions. This is particularly true for clients with a moderate level of sleep complaints rather than syndromal insomnia [52]. However, Tai Chi Chuan can be learned and practiced in a relaxed manner both indoors and outdoors and can even be directed by a video tutorial at home. Second, people with poor sleep quality may stay motivated with Tai Chi Chuan training as it can be practiced in a group-based form and offers social support and enjoyment [81]. Finally, Tai Chi Chuan provides not only mental health improvements (e.g., dementia, depression, and insomnia) but also positive effects on various common diseases (e.g., osteoarthritis, Parkinson's disease, and chronic obstructive pulmonary disease) [85]. Despite the considerable positive benefits discussed, whether Tai Chi Chuan to some extent is superior to universally recommended CBT is still open for dispute until further studies validate the current findings.

5.3. Analysis of High Heterogeneity among Included Trials. A moderate to high level of heterogeneity was observed either in our review or in the previous systematic reviews and meta-analyses [29, 32-34]. Although we applied more restrictive search strategies that only recruited RCTs with PSQI as the outcome measurement, the heterogeneity of meta-analysis results did not decline as more Chinese and English articles and several conditions of participants were involved. Two preplanned subgroup analyses seemed to explain the high heterogeneity to some degree. On the one hand, the heterogeneity decreased to a moderate level $\left(I^{2}=64.6 \%, P=0.038\right)$ among studies recruiting healthy Americans in the subgroup of the geographic population. On the other hand, the disease-based subgroup eliminated the heterogeneity among studies that recruited participants with stroke, fibromyalgia, and heart disease, respectively $\left(I^{2}=0 \%\right)$. In order to examine potential contributors to the severe heterogeneity among trials, we then performed the meta-regression of the ESs for global PSQI scores, and several moderators of the sleep quality responses to Tai Chi Chuan were identified: (a) sleep quality promotions were most considerable among trials that prescribed Tai Chi exercise for 60 to $90 \mathrm{~min}$ per session, followed by trials that practiced Tai Chi for 30 to $60 \mathrm{~min}$ per session; (b) sleep enhancements were more effective among trials that recruited the Asian population than trials with the American population; (c) sleep quality improvements were more significant in trials that were ranked in the middle tier of methodological quality than trials that ranked in the highest tier of methodological quality. Interestingly, among all the tested moderators, physical condition (fitness and illness) and disease (good health, stroke, fibromyalgia, cancer, depression, arthritis, chronic kidney disease, and heart disease) 
did not manifest any statistical significance in the regression model. This could possibly be attributed to the fact that there were so few trials in each disease (stroke: $k=2$, fibromyalgia: $k=4$, cancer: $k=3$, chronic kidney disease: $k=1$, heart disease: $k=2$, arthritis: $k=1$, and depression: $k=2$ ) which led to insufficient statistical power for difference detection. Instead, the methodological quality of studies was the largest source of heterogeneity among the studies reviewed, the exact explanation of which was unclear. Nevertheless, we observed that trials with moderate and lower methodological quality tended to have been implemented in Asia $(\rho=-0.743, P<0.001)$ and consisted of a smaller sample size ( $\rho=0.543, P=0.005)$ (see Supplementary Data Table S2). More notably, the results of the meta-regression analyses demonstrated that the geographic population was also a cofounder of the heterogeneity identified among studies. This finding suggests that the sleep quality of Asian, American, and European populations may respond to Tai Chi Chuan in different ways. Therefore, it has been identified that the integrated physical, behavioral, spiritual, and psychosocial elements of Tai Chi exercise warrant modification and localization according to the specific constitutions of different geographic populations. Finally, intensity(session time), frequency, and treatment duration were noted to be the three main components of Tai Chi Chuan intervention that were the most influential moderators in our review. However, our analysis of the current data extracted from RCTs revealed that the Tai Chi Chuan training intensity was the only component found that affected sleep quality response to Tai Chi Chuan exercise. Therefore, when physicians and therapists prescribe Tai Chi Chuan exercise for their clients with poor sleep quality or insomnia, training intensity should be emphasized as the first element to be determined according to the health status of clients. Subsequently, the frequency and treatment duration of exercise can be adjusted accordingly based on intensity.

5.4. Strengths: Comparison to Previous Systematic Reviews and Meta-Analyses. Some results obtained by this review were consistent with several previous systematic reviews and meta-analyses in that Tai Chi Chuan may be considered beneficial for insomnia [29-34]. However, only two of the previous systematic reviews and meta-analyses regarded Tai Chi Chuan as their main study subject $[33,34]$, and one of the two studies was directed and supervised by one member $\mathrm{Xu}$ ) of our current workgroup [34]. The four remaining systematic reviews and meta-analyses aimed to investigate the evidence of meditative movement interventions or mind-body therapies on sleep quality [29-32]. Two of the four systematic reviews utilized the meta-analysis [29, 32] while the other two used the narrative synthesis [30, 31]. There were nine out of 14 included trials that were related to Tai Chi Chuan intervention in the review of $\mathrm{Wu}$ et al. [29], seven out of 112 in the review of Neuendorf et al. [30], nine out of 17 in the review of Wang et al. [31], and 12 out of 49 in the review of Wang et al. [32], respectively.

This current research remarkably differs from the previous one. The latest systematic review and meta-analysis conducted by Wang et al. [32] was discussed as an example to obtain more detailed information concerning the differences between previous systematic reviews and this review. Wang et al. [32] estimated the impact of mind-body therapies on insomnia in both aged healthy and clinical populations. However, some disadvantages were observed which are listed as follows: (a) the reviewed studies involved interventions of Tai Chi Chuan, yoga, meditation, Qigong, and Qigong Tai Chi but only 12 articles were related to Tai Chi Chuan (total: 40); (b) Qigong Tai Chi only consists of the practice of Tai Chi Chuan; thus, Qigong Tai Chi and Tai Chi Chuan were naturally the same and did not warrant a separate evaluation; (c) no pooled results of Tai Chi exercise were reported since no tailored subgroup analysis of Tai Chi Chuan was performed independently; (d) no planned subgroup analysis was directed in terms of the different kinds of physical conditions or diseases; this typically contributed to substantial heterogeneity and sometimes inaccurate results; and (e) Tai Chi Chuan is a traditional Chinese martial art but only English studies were included. Given such limitations, the effects of Tai Chi Chuan on sleep quality remained vague and inconclusive.

On the contrary, the current work recruited a total of 25 updated RCTs published in English and Chinese. We focused on assessing the impact of Tai Chi Chuan on sleep quality and conducted subgroup analyses according to the various physical conditions and geographic populations. Additionally, meta-regression was implemented as a means to explore the possible sources of the high level of heterogeneity. Meanwhile, additive models were also applied to identify the optimal combination of Tai Chi Chuan elements on sleep quality. Moreover, we employed sensitivity analysis methods to test the stability of the meta-analysis results and examined the publication bias in both visual and statistical manners. More importantly, this review compiled the clinically relevant elements of Tai Chi Chuan intervention, which could guide future studies. As a result of all such factors, our review provided more precise meta-analysis results and stronger evidence for Tai Chi Chuan on subjective sleep quality in comparison with previous reviews.

5.5. Limitations. The primary limitations of the current review are listed as follows: (a) high heterogeneity existed among included RCTs; this is likely attributed to the fact that data were extracted from participants with various populations and physical conditions; (b) there was an insufficient quantity of reviewed trials in some disease categories (heart disease, depression, arthritis, stroke, and cancer); this may have contributed to weak evidence or even meaningless conclusions; (c) the included trials consisted of inconsistent Tai Chi Chuan components (training intensity, frequency, and treatment duration) which may have influenced the pooled results.

Another limitation is that we choose the global PSQI scores as the sole parameter for sleep quality outcomes. It should be noted that PQSI is a widely translated and employed self-reporting questionnaire, which plays a significant role in sleep status evaluation in both research and 
medical settings $[78,86]$. It contains multiple notable advantages such as high cost-effectiveness, easy implementation, and high patient compliance allowing clinicians and researchers to save considerable time [37]. As a result, PSQI is often employed in sleep-related studies. However, Manzar et al. [89] found the multiple PSQI factor structures for sleep quality appraisal in both clinical and nonclinical settings to be doubtful and in possible need of further validation. Besides, sleep quality changes in insomniacs comorbid with psychiatric and medical conditions which could also be embodied through other disease-specific approaches (e.g., applying an electrocardiogram-based spectrogram method to evaluate sleep quality in patients with chronic heart disease) [90]. Nevertheless, we could not assess these disease-specific outcomes as they are not universally utilized in the RCTs with regard to Tai Chi exercise for sleep quality.

Although the meta-regression and additive models identified a clinically meaningful proportion of each factor in ESs, a large amount of heterogeneity of Tai Chi Chuan sleep-promoting effects unsurprisingly remained unexplained. In addition to the factor of geographic population, training intensity, and methodological quality, eight other possible sources of heterogeneity were examined and found to be not statistically significant on the sleep quality responses to Tai Chi exercise (see Supplementary Data Table S1). Otherwise, some researchers from the reviewed Tai Chi Chuan RCTs might have omitted some detailed intervention information (e.g., when was Tai Chi Chuan practiced during the day?) in their studies which were likely to have some objective reasons (e.g., space limitation). This concern could militate against the implementation of our data extraction and analysis. For instance, we were unable to investigate some clinically relevant moderators due to inadequate disclosure, such as the Tai Chi Chuan postures $(k=4,16.0 \%)$ and the assessment of participant fatigue after Tai Chi Chuan training $(k=3,12.0 \%)$. Consequently, it is necessary for our current results to be interpreted with caution in the context of the aforementioned limitations.

\section{Conclusion}

In conclusion, this present review suggests that Tai Chi Chuan exercise has a moderate beneficial impact on subjective sleep quality among adults. Sleep quality enhancements of this level rival those reported from CBT, the initial nonpharmacological therapy for adults with insomnia. It also reflects that the current authoritative guidelines may include Tai Chi Chuan as a recommended choice of complementary and alternative treatments. Additionally, we discovered that when practiced between 60 and 90 min per session, Tai Chi Chuan can elicit the most considerable sleep-promoting effects among Asians. However, further studies are necessary as a means to optimize other Tai Chi Chuan components in accordance with the particular characteristics and aspects in different populations. The evidence of the effects of Tai Chi exercise on sleep quality among the clinical population appears to be weak. Consequently, more comprehensive and randomized trials of Tai
Chi Chuan for subjective sleep quality need to be implemented in order to ascertain the present findings.

\section{Abbreviations \\ CBT: Cognitive behavioral therapy \\ CNKI: China National Knowledge Infrastructure \\ ES: $\quad$ Effect size \\ PEDro: $\quad$ Physiotherapy Evidence Database scale \\ PRISMA: Preferred Reporting Items for Systematic Reviews and Meta-Analyses \\ PROSPERO: International Prospective Register of Systematic Reviews \\ PSQI: $\quad$ Pittsburgh Sleep Quality Index \\ RCTs: $\quad$ Randomized controlled trials \\ SD: $\quad$ Standard deviation \\ SMD: $\quad$ Standardized mean difference.}

\section{Data Availability}

All data compiled or analyzed during this study are included in this published article.

\section{Disclosure}

The funders had no role in the study design, data collection and analysis, and publication or development of the manuscript.

\section{Conflicts of Interest}

The authors declare that they have no conflicts of interest.

\section{Authors' Contributions}

GX, YM, and YS conceived this study. YS, CW, JZ, and YG were responsible for literature search, data screening, data extraction, and quality assessment. YM and GX supervised the study. YS and JZ were involved in the process of data calculation and interpretation. YS wrote the manuscript, and YM, HY, and GX revised it. All authors approved the final paper.

\section{Acknowledgments}

The authors like to express their gratitude to Professor $\mathrm{Xi}$ Huang (the "Outstanding Youth" at Nanjing University of Chinese Medicine) for his kind financial support. They also appreciate the native speaker Tina who helped us with English language editing. This study was supported by a project funded by the Priority Academic Program Development of Jiangsu Higher Education Institutions (Integration of Chinese and Western Medicine \& Nursing with nos. 2019YSHL154 and 2019YSHL151), the Postgraduate Research \& Practice Innovation Program of Jiangsu Province (no. KYCX20_1462), the National Natural Science Foundation of China (no. 81973878), the Jiangsu Natural Science Foundation (BK20180167), and the Wuxi Municipal Health Planning Commission's Science and Education Project (QNRC042). 


\section{Supplementary Materials}

Table S1: meta-regression-the eight covariables that did not show any statistical significance. Table S2: Spearman's correlation analysis-the relationship between each factor and methodological quality. (Supplementary Materials)

\section{References}

[1] B. C. Clawson, J. Durkin, and S. J. Aton, "Form and function of sleep spindles across the lifespan," Neural Plasticity, vol. 2016, Article ID 6936381, 16 pages, 2016.

[2] C. M. Morin, C. L. Drake, A. G. Harvey et al., "Insomnia disorder," Nature Reviews Disease Primers, vol. 1, no. 1, pp. 1-18, 2015.

[3] A. Huffington, "The sleep revolution: transforming your life, one night at a time," Harmony, Islamabad, Pakistan, 2016.

[4] S. P. Becker, M. A. Jarrett, A. M. Luebbe, A. A. Garner, G. L. Burns, and M. J. Kofler, "Sleep in a large, multi-university sample of college students: sleep problem prevalence, sex differences, and mental health correlates," Sleep Health, vol. 4, no. 2, pp. 174-181, 2018.

[5] M. M. Ohayon and C. F. Reynolds III, "Epidemiological and clinical relevance of insomnia diagnosis algorithms according to the DSM-IV and the International Classification of Sleep Disorders (ICSD)," Sleep Medicine, vol. 10, no. 9, pp. 952-960, 2009.

[6] L. Plantinga, M. N. Rao, and D. Schillinger, "Prevalence of self-reported sleep problems among people with diabetes in the United States, 2005-2008," Preventing Chronic Disease, vol. 9, p. E76, 2012.

[7] F. C. Baker, M. De Zambotti, I. M. Colrain, and B. Bei, "Sleep problems during the menopausal transition: prevalence, impact, and management challenges," Nature and Science of Sleep, vol. 10, pp. 73-95, 2018.

[8] X. L. Cao, S. B. Wang, B. L. Zhong et al., "The prevalence of insomnia in the general population in China: a meta-analysis," PLoS One, vol. 12, no. 2, Article ID e0170772, 2017.

[9] O. Itani, Y. Kaneita, T. Munezawa et al., "Nationwide epidemiological study of insomnia in Japan," Sleep Medicine, vol. 25, pp. 130-138, 2016.

[10] C. M. Morin, M. LeBlanc, L. Bélanger, H. Ivers, C. Mérette, and J. Savard, "Prevalence of insomnia and its treatment in Canada," The Canadian Journal of Psychiatry, vol. 56, no. 9, pp. 540-548, 2011.

[11] C. A. B. de Lira, M. S. Andrade, M. T. de Mello, and R. L. Vancini, "Physical exercise to manage sleep problems in pediatric patients with epilepsy and ADHD," Epilepsy \& Behavior, vol. 75, pp. 271-272, 2017.

[12] P. M. Wayne and T. J. Kaptchuk, "Challenges inherent to T'ai chi research: Part I-T'ai chi as a complex multicomponent intervention," The Journal of Alternative and Complementary Medicine, vol. 14, no. 1, pp. 95-102, 2008.

[13] A. Chillag, "Tai Chi fights stress,” 2019, https://www.cnn.com/ 2017/09/05/health/tai-chi-millennials/index.html.

[14] B. H. Jacobson, C. Ho-Cheng, C. Cashel, and L. Guerrero, "The effect of T'ai Chi Chuan training on balance, kinesthetic sense, and strength," Perceptual and Motor Skills, vol. 84, no. 1, pp. 27-33, 1997.

[15] L. Vitetta, B. Anton, F. Cortizo et al., "Mind-body medicine: stress and its impact on overall health and longevity," Annals of the New York Academy of Sciences, vol. 1057, no. 1, pp. 492-505, 2005.
[16] Y. Zeng, X. Xie, and A. S. K. Cheng, "Qigong or Tai Chi in cancer care: an updated systematic review and meta-analysis," Current Oncology Reports, vol. 21, no. 6, p. 48, 2019.

[17] C. Wu, Q. Yi, X. Zheng et al., "Effects of mind-body exercises on cognitive function in older adults: a meta-analysis," Journal of the American Geriatrics Society, vol. 67, no. 4, pp. 749-758, 2019.

[18] T.-W. Xia, Y. Yang, W.-H. Li, Z.-H. Tang, Z.-R. Li, and L.-J. Qiao, "Different training durations and styles of tai chi for glucose control in patients with type 2 diabetes: a systematic review and meta-analysis of controlled trials," BMC Complementary and Alternative Medicine, vol. 19, no. 1, p. 63, 2019.

[19] D. Lyu, X. Lyu, Y. Zhang et al., "Tai Chi for stroke rehabilitation: a systematic review and meta-analysis of randomized controlled trials," Frontiers in Physiology, vol. 9, p. 983, 2018.

[20] Y.-l. Yang, Y.-h. Wang, S.-r. Wang, P.-s. Shi, and C. Wang, "The Effect of Tai Chi on cardiorespiratory fitness for coronary disease rehabilitation: a systematic review and metaanalysis," Frontiers in Physiology, vol. 8, p. 1091, 2018.

[21] X. Ren, Y. Li, X. Yang et al., "The effects of tai chi training in patients with heart failure: a systematic review and metaanalysis," Frontiers in Physiology, vol. 8, p. 989, 2017.

[22] T. H. Chow, B. Y. Lee, A. B. F. Ang, V. Y. K. Cheung, M. M. C. Ho, and S. Takemura, "The effect of Chinese martial arts Tai Chi Chuan on prevention of osteoporosis: a systematic review," Journal of Orthopaedic Translation, vol. 12, pp. 74-84, 2018.

[23] Z. Lian, L. Yang, Y. Bian et al., "Effects of Tai chi on adults with essential hypertension in China: a systematic review and meta-analysis," European Journal of Integrative Medicine, vol. 12, pp. 153-162, 2017.

[24] A. Hall, B. Copsey, H. Richmond et al., "Effectiveness of tai chi for chronic musculoskeletal pain conditions: updated systematic review and meta-analysis," Physical Therapy, vol. 97, no. 2, pp. 227-238, 2017.

[25] H. C. Shen and S. E. Guo, "Effects of Tai Chi on exercise tolerance, quality of life, and health status in patients with chronic obstructive pulmonary disease-a systematic review," Taiwan Gong Gong Wei Sheng Za Zhi, vol. 35, no. 1, p. 39, 2016.

[26] Z. G. Huang, Y. H. Feng, Y. H. Li, and C.-S. Lv, "Systematic review and meta-analysis: tai Chi for preventing falls in older adults," BMJ Open, vol. 7, no. 2, Article ID e013661, 2017.

[27] W. D. Chang, S. Chen, C. L. Lee, H.-Y. Lin, and C. L. Lai, "The effects of tai chi chuan on improving mind-body health for knee osteoarthritis patients: a systematic review and metaanalysis," Evidence-Based Complementary and Alternative Medicine, vol. 2016, Article ID 1813979, 10 pages, 2016.

[28] L. Zou, J. Han, C. Li et al., "Effects of Tai Chi on lower limb proprioception in adults aged over 55: a systematic review and meta-analysis," Archives of Physical Medicine and Rehabilitation, vol. 100, no. 6, pp. 1102-1113, 2019.

[29] W.-w. Wu, E. Kwong, X.-y. Lan, and X.-y. Jiang, "The effect of a meditative movement intervention on quality of sleep in the elderly: a systematic review and meta-analysis," The Journal of Alternative and Complementary Medicine, vol. 21, no. 9, pp. 509-519, 2015.

[30] R. Neuendorf, H. Wahbeh, I. Chamine et al., "The effects of mind-body interventions on sleep quality: a systematic review," Evidence-Based Complementary and Alternative Medicine, vol. 2015, Article ID 902708, 17 pages, 2015.

[31] F. Wang, O. Eun-Kyoung Lee, F. Feng et al., "The effect of meditative movement on sleep quality: a systematic review," Sleep Medicine Reviews, vol. 30, pp. 43-52, 2016. 
[32] X. Wang, P. Li, C. Pan et al., "The effect of mind-body therapies on insomnia: a systematic review and meta-analysis," Evidence-based Complementary and Alternative Medicine, vol. 2019, Article ID 9359807, 17 pages, 2019.

[33] G. Raman, Y. Zhang, V. J. Minichiello, C. M. D’Ambrosio, and C. Wang, "Tai Chi improves sleep quality in healthy adults and patients with chronic conditions: a systematic review and meta-analysis," Journal of Sleep Disorders \& Therapy, vol. 2, no. 6, 2013.

[34] S. Du, J. Dong, H. Zhang et al., "Taichi exercise for self-rated sleep quality in older people: a systematic review and metaanalysis," International Journal of Nursing Studies, vol. 52, no. 1, pp. 368-379, 2015.

[35] D. Riemann, C. Baglioni, C. Bassetti et al., "European guideline for the diagnosis and treatment of insomnia," Journal of Sleep Research, vol. 26, no. 6, pp. 675-700, 2017.

[36] A. Qaseem, D. Kansagara, M. A. Forciea, M. Cooke, and T. D. Denberg, "Management of chronic insomnia disorder in adults: a clinical practice guideline from the American college of physicians," Annals of Internal Medicine, vol. 165, no. 2, pp. 125-133, 2016.

[37] M. D. Manzar, J. A. Moiz, J. A. Moiz et al., "Validity of the Pittsburgh sleep quality index in Indian university students," Oman Medical Journal, vol. 30, no. 3, pp. 193-202, 2015.

[38] D. Moher, A. Liberati, J. Tetzlaff et al., "Preferred reporting items for systematic reviews and meta-analyses: the PRISMA statement," Annals of Internal Medicine, vol. 151, no. 4, pp. 264-269, 2009.

[39] Y. Si, C. Wang, J. Zheng, Y. Guo, G. Xu, and Y. Ma, “Tai Chi exercise for sleep problems in older adults: a protocol of systematic review and meta-analysis," Medicine, vol. 98, no. 45 , p. e17556, 2019.

[40] S. K. Bhogal, R. W. Teasell, N. C. Foley, and M. R. Speechley, "The PEDro scale provides a more comprehensive measure of methodological quality than the Jadad scale in stroke rehabilitation literature," Journal of Clinical Epidemiology, vol. 58, no. 7, pp. 668-673, 2005.

[41] N. A. de Morton, "The PEDro scale is a valid measure of the methodological quality of clinical trials: a demographic study," Australian Journal of Physiotherapy, vol. 55, no. 2, pp. 129-133, 2009.

[42] C. G. Maher, C. Sherrington, R. D. Herbert, A. M. Moseley, and M. Elkins, "Reliability of the PEDro scale for rating quality of randomized controlled trials," Physical Therapy, vol. 83, no. 8, pp. 713-721, 2003.

[43] T. P. Yamato, C. Maher, B. Koes, and A. Moseley, "The PEDro scale had acceptably high convergent validity, construct validity, and interrater reliability in evaluating methodological quality of pharmaceutical trials," Journal of Clinical Epidemiology, vol. 86, pp. 176-181, 2017.

[44] B. T. Johnson and T. B. Huedo-Medina, Meta-Analytic Statistical Inferences for Continuous Measure Outcomes as a Function of Effect Size Metric and Other Assumptions, Agency for Healthcare Research and Quality, Rockville, MD, USA, 2013.

[45] J. Cohen, Statistical Power Analysis for the Behavioral Sciences, Academic Press, Cambridge, MA, USA, 2013.

[46] J. P. T. Higgins, S. G. Thompson, J. J. Deeks et al., "Measuring inconsistency in meta-analyses," BMJ, vol. 327, no. 7414, pp. 557-560, 2003.

[47] J. M. Northey, N. Cherbuin, K. L. Pumpa, D. J. Smee, and B. Rattray, "Exercise interventions for cognitive function in adults older than 50: a systematic review with meta-analysis," British Journal of Sports Medicine, vol. 52, no. 3, pp. 154-160, 2018.
[48] S. Colcombe and A. F. Kramer, "Fitness effects on the cognitive function of older adults," Psychological Science, vol. 14, no. 2, pp. 125-130, 2003.

[49] F. Li, K. J. Fisher, P. Harmer, D. Irbe, R. G. Tearse, and C. Weimer, "Tai chi and self-rated quality of sleep and daytime sleepiness in older adults: a randomized controlled trial," Journal of the American Geriatrics Society, vol. 52, no. 6, pp. 892-900, 2004.

[50] B. Frye, S. Scheinthal, T. Kemarskaya, and R. Pruchno, "Tai chi and low impact exercise: effects on the physical functioning and psychological well-being of older people," Journal of Applied Gerontology, vol. 26, no. 5, pp. 433-453, 2007.

[51] C. Han, "The influence of Taiji softball on the aged persons' sleep quality and emotion," Fujian Ti Yu Ke Ji, vol. 2, pp. 21-22, 2008.

[52] M. R. Irwin, R. Olmstead, and S. J. Motivala, "Improving sleep quality in older adults with moderate sleep complaints: a randomized controlled trial of Tai Chi Chih," Sleep, vol. 31, no. 7, pp. 1001-1008, 2008.

[53] R. Liu, "Influence of 8-week shadowboxing exercise on the indexes for evaluating sleep behavior in elderly people," Zhongguo Lao Nian Bao Jian Yi Xue, vol. 8, no. 5, pp. 26-27, 2010.

[54] C. Wang, C. H. Schmid, R. Rones et al., "A randomized trial of tai chi for fibromyalgia," New England Journal of Medicine, vol. 363, no. 8, pp. 743-754, 2010.

[55] W. Wang, M. Sawada, Y. Noriyama et al., "Tai Chi exercise versus rehabilitation for the elderly with cerebral vascular disorder: a single-blinded randomized controlled trial," Psychogeriatrics, vol. 10, no. 3, pp. 160-166, 2010.

[56] H. Hosseini, M. F. Esfirizi, S. M. Marandi et al., "The effect of Ti Chi exercise on the sleep quality of the elderly residents in Isfahan, Sadeghieh elderly home," Iranian Journal of Nursing and Midwifery Research, vol. 16, no. 1, pp. 55-60, 2011.

[57] K. D. Jones, C. A. Sherman, S. D. Mist, J. W. Carson, R. M. Bennett, and F. Li, "A randomized controlled trial of 8form Tai chi improves symptoms and functional mobility in fibromyalgia patients," Clinical Rheumatology, vol. 31, no. 8, pp. 1205-1214, 2012.

[58] M. H. Nguyen and A. Kruse, "A randomized controlled trial of Tai chi for balance, sleep quality and cognitive performance in elderly Vietnamese," Clinical Interventions in Aging, vol. 7, pp. 185-190, 2012.

[59] M. R. Irwin, R. Olmstead, C. Carrillo et al., "Cognitive behavioral therapy vs. Tai Chi for late life insomnia and inflammatory risk: a randomized controlled comparative efficacy trial," Sleep, vol. 37, no. 9, pp. 1543-1552, 2014.

[60] R. E. Taylor-Piliae, T. M. Hoke, J. T. Hepworth, L. D. Latt, B. Najafi, and B. M. Coull, "Effect of tai chi on physical function, fall rates and quality of life among older stroke survivors," Archives of Physical Medicine and Rehabilitation, vol. 95, no. 5, pp. 816-824, 2014.

[61] L. K. Larkey, D. J. Roe, K. L. Weihs et al., "Randomized controlled trial of qigong/tai chi easy on cancer-related fatigue in breast cancer survivors," Annals of Behavioral Medicine, vol. 49, no. 2, pp. 165-176, 2015.

[62] Y. Liu, "Impacts of an 18-week Taiji ball exercise on the sleeping quality, mental condition and life contentment of elderly people," Nanjing Ti Yu Xue Yuan Xue Bao, vol. 29, no. 3, pp. 116-121, 2015.

[63] Q. Liu, "Effects of 16 weeks Tai Chi exercise and stop practice 8 weeks impact on mental health of older women," Shandong Ti Yu Xue Yuan Xue Bao, vol. 32, no. 6, pp. 99-103, 2016.

[64] S. Maddali Bongi, G. Paoletti, M. Calà, A. Del Rosso, K. El Aoufy, and S. Mikhaylova, "Efficacy of rehabilitation with tai ji 
quan in an Italian cohort of patients with fibromyalgia syndrome," Complementary Therapies in Clinical Practice, vol. 24, pp. 109-115, 2016.

[65] H. Z. R. Fan and L. Chang, "The effect of Tai Chi exercise on the state of anxiety and depression and sleep quality in hemodialysis patients," Zhongguo Xue Ye Jing Hua, vol. 15, no. 4, pp. 241-243, 2016.

[66] L. Yuan, H. Zhang, F. Zhou et al., "Effect of Tai Chi on improvement of depression, sleeping quality and quality of life in elderly patients with chronic congestive heart failure complicated with depression," Guangxi Yi Xue, vol. 38, no. 11, pp. 1547-1550, 2016.

[67] Y. Chen, X. Wang, and M. Zhang, "Effect of Tai Chi exercise on the sleep quality for older adults with coronary heart disease," An Mo Yu Kang Fu Yi Xue, vol. 8, no. 5, pp. 64-66, 2017.

[68] M. R. Irwin, R. Olmstead, C. Carrillo et al., "Tai Chi Chih compared with cognitive behavioral therapy for the Treatment of Insomnia in Survivors of Breast Cancer: a randomized, partially blinded, noninferiority trial," Journal of Clinical Oncology, vol. 35, no. 23, pp. 2656-2665, 2017.

[69] J. Lv, L. Huang, X. Wu et al., "Effect of Tai Ji Quan training on self-reported sleep quality in elderly Chinese women with knee osteoarthritis: a randomized controlled trial," Sleep Med, vol. 33, pp. 70-75, 2017.

[70] J. L. McQuade, S. Prinsloo, D. Z. Chang et al., "Qigong/tai chi for sleep and fatigue in prostate cancer patients undergoing radiotherapy: a randomized controlled trial," Psycho-Oncology, vol. 26, no. 11, pp. 1936-1943, 2017.

[71] T. Rao and H. Ni, "Effects of Taijiquan on sleep and emotion in patients with depression-related insomnia," Zhongguo Chu Ji Wei Sheng Bao Jian, vol. 32, no. 1, pp. 66-78, 2018.

[72] C. Wang, C. H. Schmid, R. A. Fielding et al., "Effect of tai chi versus aerobic exercise for fibromyalgia: comparative effectiveness randomized controlled trial," BMJ, vol. 360, p. k851, 2018.

[73] D. Zhu, G. Dai, D. Xu et al., "Long-term effects of Tai Chi intervention on sleep and mental health of female individuals with dependence on amphetamine-type stimulants," Frontiers in Psychology, vol. 9, 2018.

[74] H. Deng and J. Wang, "The effectiveness of Tai Chi and traditional music on psychological health and sleep quality for community-dwelling older people," Zhongguo Lao Nian Bing Xue Za Zhi, vol. 33, no. 17, pp. 4229-4230, 2013.

[75] L. J. Kong, R. Lauche, P. Klose et al., "Tai chi for chronic pain conditions: a systematic review and meta-analysis of randomized controlled trials," Scientific Reports, vol. 6, no. 1, p. 25325, 2016.

[76] R. Jahnke, L. Larkey, C. Rogers, J. Etnier, and F. Lin, “A comprehensive review of health benefits of qigong and tai chi," American Journal of Health Promotion, vol. 24, no. 6, pp. e1-e25, 2010.

[77] P. Montgomery and J. A. Dennis, "Physical exercise for sleep problems in adults aged 60+," Cochrane Database of Systematic Reviews, no. 4, , 2002.

[78] H. W. L. Koh, R. B. T. Lim, K. S. Chia, and W. Y. Lim, "The Pittsburgh Sleep Quality Index in a multi-ethnic Asian population contains a three-factor structure," Sleep and Breathing, vol. 19, no. 4, pp. 1147-1154, 2015.

[79] P.-Y. Yang, K.-H. Ho, H.-C. Chen, and M.-Y. Chien, "Exercise training improves sleep quality in middle-aged and older adults with sleep problems: a systematic review," Journal of Physiotherapy, vol. 58, no. 3, pp. 157-163, 2012.
[80] A. van Straten, T. van der Zweerde, A. Kleiboer, P. Cuijpers, C. M. Morin, and J. Lancee, "Cognitive and behavioral therapies in the treatment of insomnia: a meta-analysis," Sleep Medicine Reviews, vol. 38, pp. 3-16, 2018.

[81] N. P. Gothe and B. J. Kendall, "Barriers, motivations, and preferences for physical activity among female African American older adults," Gerontology and Geriatric Medicine, vol. 2, 2016.

[82] L. Yang, "Effect of "morning tai chi, evening baduan on sleep quality of community type 2 diabetes patients with insomnia," Hu Li Shi Jian Yan Jiu, vol. 10, no. 1, pp. 16-18, 2013.

[83] M. R. Irwin, R. Olmstead, and M. N. Oxman, "Augmenting immune responses to varicella zoster virus in older adults: a randomized, controlled trial of Tai Chi," Journal of the American Geriatrics Society, vol. 55, no. 4, pp. 511-517, 2007.

[84] J. Q. Wu, E. R. Appleman, R. D. Salazar, and J. C. Ong, "Cognitive behavioral therapy for insomnia comorbid with psychiatric and medical conditions," JAMA Internal Medicine, vol. 175, no. 9, pp. 1461-1472, 2015.

[85] P. Huston and B. McFarlane, "Health benefits of tai chi: what is the evidence?" Canadian Family Physician, vol. 62, no. 11, pp. 881-890, 2016.

[86] M. Salahuddin, T. T. Maru, A. Kumalo et al., "Validation of the Pittsburgh sleep quality index in community dwelling Ethiopian adults," Health and Quality of Life Outcomes, vol. 15, no. 1, p. 58, 2017.

[87] Y.-F. C. Chao, S.-Y. Chen, C. Lan, and J.-S. Lai, “The cardiorespiratory response and energy expenditure of Tai-ChiQui-Gong," The American Journal of Chinese Medicine, vol. 30, no. 4, pp. 451-461, 2002.

[88] C. M. Morin, R. R. Bootzin, D. J. Buysse, J. D. Edinger, C. A. Espie, and K. L. Lichstein, "Psychological and behavioral treatment of insomnia: update of the recent evidence (1998-2004)," Sleep, vol. 29, no. 11, pp. 1398-1414, 2006.

[89] M. D. Manzar, A. S. BaHammam, U. A. Hameed et al., "Dimensionality of the Pittsburgh sleep quality index: a systematic review," Health and Quality of Life Outcomes, vol. 16, no. 1, p. 89, 2018.

[90] G. Y. Yeh, J. E. Mietus, C.-K. Peng et al., "Enhancement of sleep stability with Tai Chi exercise in chronic heart failure: preliminary findings using an ECG-based spectrogram method," Sleep Medicine, vol. 9, no. 5, pp. 527-536, 2008. 